\title{
Concurrent Identification of Impact Location and Force Magnitude on a Composite Panel
}

\author{
Hamed Kalhori, , ,*, Mehrisadat Makki Alamdari ${ }^{3}$, Bing Li $^{4}$, Ben Halkon', Seyedeh Marzieh Hosseini ${ }^{1}$, \\ Lin $\mathrm{Ye}^{5}$, Zheng $\mathrm{Li}^{6}$ \\ ${ }^{1}$ School of Mechanical and Mechatronic Engineering, University of Technology Sydney, Ultimo, NSW 2007, Australia \\ ${ }^{2}$ Department of Mechanical Engineering, Faculty of Engineering, Bu-Ali Sina University, Hamedan, Iran \\ ${ }^{3}$ School of Civil and Environmental Engineering, University of New South Wales, NSW 2052, Australia \\ ${ }^{4}$ School of Aeronautics, Northwestern Polytechnical University, Xi'an, Shaanxi, 710072, China \\ ${ }^{5}$ School of Aerospace, Mechanical and Mechatronic Engineering, The University of Sydney, NSW 2006, Australia \\ ${ }^{6}$ Department of Mechanics and Aerospace Engineering, College of Engineering, Peking University, Beijing 100871, China
}

\section{Abstract}

Simultaneous estimation of both the location and force history of an impact applied on a lattice truss core sandwich panel is inversely carried out utilising velocity signals collected by means of a scanning laser Doppler vibrometer. The algorithm assumes that several impact forces are exerted concurrently on a number of specified locations on a panel, provided that the magnitude of all impact forces but one is actually equal to zero. This condition equates to a scenario where an impact occurs at only one location. The purpose is therefore to detect the actual impact location among all potential

\footnotetext{
* To whom all correspondence should be addressed

Email: hamed.kalhori@uts.edu.au, Tel: +61-02-9351 4798
} 
locations, together with its force history, through minimising error functions. Two algorithms, the oneto-one (even-determined) approach and the superposition approach, are considered. The one-to-one approach solves the reconstruction problem independently for each pair of impact and measurement points. However, in the superposition approach, the impact forces at all potential locations are concurrently reconstructed through a single matrix equation. It is shown that the one-to-one approach fails to detect the true impact location while the superposition approach recognises the actual impact location based on some qualitative evaluating criteria. Adopting the superposition approach, for a problem with four possible impact locations, two scenarios one with four and one with twelve measurement points, are investigated. It is observed that the additional measurement points do not necessarily enhance the efficiency and accuracy of the proposed method. It is found that different arrangements of measuring points lead to identification of the location and the magnitude of the impact force, though the use of four evenly distributed measurement points seems to be most effective in simultaneous identification of the location and magnitude of the impact force. Further, a quantitative index based on the concept of similarity search for time-series using wavelet transformation is proposed and it is demonstrated that the index can successfully identify the true impact force location in a fully automated way.

Keywords: Composite; Impact Force Identification; Inverse Algorithm; Similarity Searching; Wavelet Transform.

\section{Introduction}

Over the past several years, there has been a great deal of attention in carbon fibre reinforced composite sandwich panels with lattice truss cores since these structures have potential multifunctional advantages benefiting from weight efficiency and high specific stiffness [1-3]. Lattice truss structures with cellular core topologies including tetrahedral lattice truss, pyramidal lattice truss, lattice block, 
octet-truss, and 3D-kagome present stiffnesses and strengths which scale linearly with respect to their relative density [1]. This makes such lattice structures at low relative densities much stiffer and stronger compared to their foam core same material equivalents, making them more suitable candidates for aerospace applications [4]. Investigation of the mechanical properties of lattice truss core sandwich panels under out-of-plane and in-plane loadings is therefore of high potential significance.

Exploration of structural failure caused by environmental low-velocity impacts is of great importance in aerospace structures, since these structures are often subjected to out-of-plane loads both during the manufacturing processes and within operation [5]. Impact can produce drastic damage leading to degradation in the stiffness, integrity and strength of lightweight composite structures. For speedy maintenance, repair and retrofitting, information as to the incidence of impact, including its location and magnitude, is of vital significance in measuring probable damage.

Direct measurement of an impact force using instrumented devices such as force transducers is only practically achievable when the location of the impact is known in advance. Indirect estimation of the dynamic force utilising structural responses captured distant from the force location therefore has been widely used $[6,7]$. Comprehensive determination of the impact is achieved by accurate identification of both its location and the force history, i.e. magnitude. The reconstruction procedure for the impact force time history can be carried out by deconvolving the recorded response signals from the transfer function of the structure [8].

Generally, deconvolution can be performed in either the frequency domain or in the time domain. Conventional methods for deconvolution, such as Gaussian elimination or the standard inverse, can lead to unsteady results. The more stable least-squares method has been implemented to address the problem [8]. However, the high sensitivity of such systems to uncertainties such as noise and measurement errors can result in a totally distorted result and the system must, therefore, be regularised $[9,10]$. 
Various regularisation techniques have been established and used by researchers. Truncated singular value decomposition (TSVD), Tikhonov and conjugate gradient (CG) methods have been widely adopted for regularisation of inverse problems for impact force determination [9].

Many methods have been put forward for identifying the location of the impact force. Triangulation utilising wave signals collected at different (three or more) locations of a structure is the most common method [11]. For this method to be applicable, wave speed through the structure must first be determined. In composite materials, normally anisotropic, wave speed is directionally dependent, complicating this process. Triangulation combined with optimisation methods has been developed to attempt to remedy this challenge with some success $[12,13]$. Impact location has also been estimated by minimising the difference between simulated and measured responses using optimisation algorithms $[14,15]$. A further strategy, cosine similarity searching has also been introduced to identify the impact location [16].

In this study, both the force history and location of an impact exerted on a lattice truss core composite sandwich panel are identified using deconvolution in the time domain. A certain number of impact forces are considered to be concurrently applied at a number of given locations on a panel, but the magnitude of all the impact forces except one is set to zero. The impression behind this scheme is that an impact has occurred at only one of the potential locations. Two approaches for solution of the problem are proposed: the one-to-one approach and the superposition approach. It is shown that the one-to-one approach implementing the deconvolution between every measurement point and impact location fails to determine the actual impact location. The superposition approach is then established on deconvolution of a matrix equation which includes the linear superposition of responses due to impact forces at different locations. A pseudo-inverse method together with truncated singular value decomposition are used to solve the least-squares problem. The impact forces are then reconstructed concurrently at all potential locations and the true location is identified based on qualitative and quantitative assessment criteria. Two strategies, namely over-determined and even-determined 
approaches, are investigated for a given number of possible impact locations. Finally, the effects of the number of measurement points and their arrangements on the algorithm are studied. It should be noted that the effect of material uncertainty has been ignored in this study.

\section{Impact identification strategy}

In a linear system, the dynamic response $R$ recorded at point $x$ due to a force exerted on location $y$ is usually expressed by a convolution integral as $[17,18]$

$$
r(x, t)=\int_{0}^{t} H(x, y, t-\tau) f(y, \tau) d \tau
$$

where $H$ is the corresponding transfer function in the time domain, which can be extracted using an inverse algorithm [19]. The convolution integral is discretised and expressed as

$$
\boldsymbol{r}=\boldsymbol{H} \boldsymbol{f}
$$

where $H$ is a lower triangular matrix

$$
H=\left[\begin{array}{ccccc}
h_{1} & 0 & \ldots & \ldots & 0 \\
h_{2} & h_{1} & \ddots & \ddots & \vdots \\
h_{3} & h_{2} & \ddots & \ddots & \vdots \\
\vdots & \vdots & \ddots & \ddots & 0 \\
h_{p} & h_{p-1} & \ldots & \ldots & h_{1}
\end{array}\right]
$$

where $h_{i}(i=1 \ldots p)$ is the value of the transfer function at time $t_{i}=i . \Delta t$, and $p$ is the number of samples.

The transfer function $\boldsymbol{H}$ is essentially defined as the structural response to a unit impulse representing the dynamic characteristics of the structure. When several impact forces $f_{i}(i=1 \ldots M)$ are simultaneously applied at different locations of a structure, the corresponding response signal at a specified measuring point $r_{j}(j=1 \ldots N)$ is a superposition of the responses produced by each individual force as 


$$
r_{j}=\sum_{i=1}^{M} H_{j}^{i} f_{i}
$$

where $H_{j}^{i}$ is the transfer function between location $i$ and measurement point $j$. For a set of measurement points, Eq. (4) will be

$$
\left[\begin{array}{l}
r_{1} \\
r_{2} \\
\vdots \\
r_{N}
\end{array}\right]=\left[\begin{array}{cccc}
H_{1}^{1} & H_{1}^{2} & \cdots & H_{1}^{M} \\
H_{2}^{1} & H_{2}^{2} & \cdots & H_{2}^{M} \\
\vdots & \vdots & \vdots & \vdots \\
H_{N}^{1} & H_{N}^{2} & \cdots & H_{N}^{M}
\end{array}\right]\left[\begin{array}{c}
f_{1} \\
f_{2} \\
\vdots \\
f_{M}
\end{array}\right]
$$

where $M$ and $N$ denote the number of impact locations and measurement points, respectively. For brevity, Eq. (5) is represented by $r=H f$. The least-squares approach is used to solve the error function for $f$ as

$$
\min (r-H f)^{T}(r-H f)
$$

where $T$ denotes the transpose of the matrix. A solution of this minimisation is acquired based on singular value decomposition of $H\left(H=U S V^{T}\right)$ and using the pseudo-inverse method as

$$
f=V S^{\ell} U^{T} r
$$

where $V$ and $U$ are square orthonormal matrices, $S^{\ell}$ is a diagonal matrix whose elements $1 / s_{i} \quad\left(1 / s_{1} \geq 1 / s_{2} \geq \ldots \geq 1 / s_{p}\right)$ are reciprocals of singular values of $H$ in which $1 / 0$ is set to zero. It should be noted that the singular values of matrix $S^{\ell}$ are denoted by $s_{\mathrm{i}}$.

Inevitable disseminated noise and measurement errors in the system, together with discretisation of the convolution integral, typically render the system ill-conditioned. Consequently, the solution obtained from Eq. (7) is typically unstable and insufficient. This problem can be mitigated by replacing the matrix $H$ by a nearby less ill-conditioned matrix. To that end, truncation of the singular values of matrix $H$, normally referred to as the TSVD method, is implemented up to a specific rank $d$. This replacement is also referred to as spectral cut-off regularisation [20]. $H$ is given by 


$$
H=U S_{d} V^{T}
$$

where $\operatorname{rank}\left(S_{d}\right) \leq \operatorname{rank}(S)$ and

$$
S_{d}=\operatorname{diag}\left[s_{1}, s_{2}, \ldots, s_{d}, 0, \ldots, 0\right] .
$$

The solution for $f$ is generally given by $f=V S_{d}^{\ell} U^{T} r$, in which the diagonal elements of $S_{d}^{\ell}$ are

$$
g_{i}=\left\{\begin{array}{ll}
1 / s_{i} & \infty>s_{i} \geq s_{d} \\
0 & s_{d}>s_{i} \geq 0
\end{array} .\right.
$$

The diagonal elements, $g_{i}$, are schematically plotted in Figure 1. The truncation index $d$ for this purpose is obtained using the generalised cross-validation method [10].

\section{Experimental set-up}

A composite sandwich panel with laminate skins and a pyramidal lattice truss core was used in this experiment, as illustrated in Figure 2. The specimen was manufactured by Harbin Institute of Technology [21] and was $260 \mathrm{~mm}$ in length, $108 \mathrm{~mm}$ in width, and $17.8 \mathrm{~mm}$ in thickness. Figure 3 shows a schematic of the specimen representing the dimensions of the panel and the unit cell. The material properties of a single lamina and a truss bar provided by the manufacturer are listed in Table 1 [22]. The layer sequence of the laminate skin was $[0 / 90]_{3 s}$. One edge of the panel was clamped and the other three edges were free.

A scanning laser Doppler vibrometer (SLDV, Polytec, PSV-400) was used to capture the out-ofplane velocity at the predefined measurement points. SLDV is a non-contact (i.e. zero mass and stiffness loading) measurement technique that can enable the rapid determination of structural vibration response with practically no upper frequency limit. LDV in general has benefits for hot, light, small and

rotating systems and/or where the context of the measurement requires high spatial resolution, high frequency range and/or remote operation [23]. In this study, the size of the specimen was fairly small 
making it challenging to attach 12 accelerometers or PZT disks to the panel. In addition, due to the low mass nature of the panel, mounting transducers will impose extra mass to the structure changing its dynamic characteristics.

To improve the quality of the reflected laser beams from the surface of the panel, patches of a special reflective tape were attached to the measurement points (Figure 2 (b)). The SLDV used in the experiment performed a sequential scanning approach in measuring the velocity at the measurement points, but it could not produce sufficiently synchronised measurements over all the points. To capture measurements over all the points as a result of an impact at a specific location, a repeatable impact was then applied at a location using a shaker (Brüel \& Kjær, Type 4810) that produced an identical templeshaped force profile. A temple-shaped force refers to a force which is similar to a half-sine signal in shape but with a wide range of frequency. Normal impact forces typically create such profile in time domain. The shaker was mounted to the rear of the panel, while the SLDV scanned the front surface. The control unit of the SLDV produced the excitation signal and a power amplifier (Brüel \& Kjær, Type 2706) amplified the signals before application to the shaker. Using a shaker instead of an instrumented hammer, as well as improving the impact repeatability, also protected the panel from possible dent damage, since it was very sensitive to impact.

The actual force profile generated by the shaker was recorded by a force transducer and the data were collected by one of the channels of the SLDV data acquisition system. Figure 4 shows the complete experimental set-up. Acquisition of the signals was triggered once the impact had been applied and was sampled at a rate of $25.6 \mathrm{kHz}$. The sampling time was set to $2.56 \mathrm{~s}$, of which $10 \%$ was pre-trigger time. Twelve measurement points and four impact locations were considered at the front and at the back of the panel, respectively, as shown in Figure 5.

The transfer function of each impact location was created by applying a reference impact force to each impact location and sequentially measuring the velocity responses at the twelve measurement points. The procedures for creating the transfer function have been detailed in previous studies [24-27]. 
An impact with unknown location and magnitude was then applied to one of these four impact locations. The study was designed to reveal the location of the impact force and its magnitude.

\section{Qualitative force identification}

Figure 6 displays a force exerted by the shaker at impact location 2, and the velocity response at point 3 is shown in Figure 7. As illustrated, a normal vibration damping in less than $0.4 s$ is represented by the structure. In this section, two different approaches, the one-to-one approach and the superposition approach, are introduced for identification of the impact force.

\subsection{One-to-one approach}

In this approach, the algorithm utilises a single measurement point to reconstruct the impact force. In other words, it solves the reconstruction problem independently for each pair of impact and measurement point in an even-determined manner (see Eq. 2). The reconstructed forces are then evaluated to identify the actual impact location. For a system with a certain number of possible impact locations and using one-to-one approach, it is ideally expected that the reconstructed impact forces at the locations other than the true impact location could indicate non-impact-like patterns since there has been no impact at those locations. As a case study, it was assumed that the impact occurred at location 3 and the velocity signal collected at single measurement point 6 was used as the vibration response. Because there are four potential locations for impact occurrence, e.g. locations 1 to 4 in Figure 5, therefore, for each location, based on the transfer function between the location and the measurement point 6 , the force signal is reconstructed using a one-to-one (even-determined) approach. For instance, the algorithm first assumes that an impact has occurred at location 1 and then reconstructs the force at that location, implementing the collected velocity response and the transfer function between impact location 1 and measurement point $6, H_{6}^{1}$. This process is repeated for impact locations 2,3 and 4 utilising $H_{6}^{2}, H_{6}^{3}$, and $H_{6}^{4}$, respectively. The reconstructed forces at locations $1,2,3$ and 4 are presented 
in Figure 8 (a)-(d), respectively. In Figure 8, 'Actual' denotes the actual force which has been applied at location 3; it is shown at all possible locations since the impact point is not known for the sake of this test case. Assuming a lack of information about the actual impact force, the reconstructed forces at the all potential locations are qualitatively very similar to each other and resemble a normal impact force. This makes the selection of the true impact location difficult. The correlation coefficients [24] between the reconstructed force and the actual impact force from $t=0 \mathrm{~ms}$ to $t=30 \mathrm{~ms}$ for locations $1,2,3$ and 4 are $0.9740,0.9796,0.9932$ and 0.8346 , respectively, showing high similarity. This phenomenon was also observed when a different measurement point was used. As a result, this method failed to determine the actual impact location using one-to-one approach. The reason why the one-to-one approach fails to identify the actual impact location could be because of the similarity between the transfer functions of different measurement points and the various impact locations due to the symmetrical and regular nature of the test specimen.

\subsection{Superposition approach}

The superposition approach is based on deconvolution of a matrix equation consisting of linear superposition of responses to impact forces at different locations (see equation 5). Unlike the one-toone approach, the impact forces at all potential locations are reconstructed concurrently through a single equation. In the analyses below, four impact forces were assumed to be applied simultaneously at the demarcated impact locations. The magnitude of all forces except one is taken as zero. The impact was applied at location 3 as a case study. Two different scenarios were considered. First, all 12 measurement points were simultaneously taken into account for calculations. The problem was thereby sufficiently over-determined as it included 12 outputs (12 measurement points) and 4 inputs (4 impact locations). Second, the velocity responses measured at 4 different measurement points were employed. In this case, the problem comprised four inputs (4 impact locations) and four outputs (4 measurement points), typically called an even-determined problem. Figure 9 displays the force applied at location 3 
and the associated velocity responses collected at measuring points 1, 4, 5 and 8 for the duration of 30 $m s$.

After reconstruction of impact forces at all possible locations, it was expected that all forces except that at the true impact location would be of very low amplitude (around zero). However, since this was not the case in most of the analyses performed in this study, some qualitative and quantitative assessment measures were proposed based on the characteristics of the reconstructed forces, including the shape and the amplitude of the first peak with respect to those of the other peaks, if applicable. It was assumed that the shape of a reconstructed impact force would resemble a nominal impact force with a normal shape (e.g. Figure 9) and the amplitude of the first peak would normally be higher than the other peaks when several peaks were present. On the other hand, from a quantitative point of view, the reconstructed force at the true impact location must have the higher peak amplitude than the forces at the other possible locations.

\subsubsection{Over-determined case}

Figure 10 illustrates the reconstructed impact forces at four potential impact locations including 4 inputs and 12 outputs. As can be seen in Figure 10 (b) and (d), the reconstructed impact forces at locations 2 and 4 have almost even magnitude except for some small oscillations, the maxima being 48 $N$ and $28 N$, respectively. These oscillations are completely different from those found with a nominal impact force that has a normal shape (e.g. Figure 9), indicating that no impact occurred at these locations, and the small fluctuations in the reconstructed forces result from noise and errors in the inverse algorithm.

As depicted in Figures 10 (a) and (c), the reconstructed forces at locations 1 and 3 consist of multiple peaks, and in particular at location 3 the first peak has a normal shape, starting almost at the ground zero. The first peak amplitude at location 3 is substantially higher than the other peaks, implying that location 3 is the actual impact location. The magnitude of the first peak at location 1 is 95 
$N$, whereas the magnitude of the first peak at location 3 is $201 N$. The second peak at both locations is around $27 \mathrm{~N}$. In fact, the magnitude of the first peak of the reconstructed force at location 1 is much lower than that of the actual impact force $(244 N)$, whereas the magnitude of the first peak at location 3 differs slightly from that of the actual force, with an error of $17.61 \%$. The correlation coefficient between the reconstructed force and the actual impact force from $t=0 \mathrm{~ms}$ to $t=30 \mathrm{~ms}$ was 0.870 for location 1 , whereas it is was 0.982 for location 3.

The results could now be further refined by decreasing the number of inputs (impact locations) from four to two (i.e. locations 1 and 3), leading to an over-determined problem with 12 outputs (measurement points). Figure 11 illustrates the reconstructed impact force at locations 1 and 3 . As seen in Figure 11 (a), the reconstructed force at location 1 is more dispersed than that in Figure 10 (a), and the maximum value of the reconstructed force is about $70 \mathrm{~N}$. The correlation coefficient between the reconstructed force and the actual force from $t=0 \mathrm{~ms}$ to $t=30 \mathrm{~ms}$ is 0.847 . Figure 11 (b) demonstrates the actual force together with the reconstructed force at location 3 . The reconstructed force and the actual force coincide and the correlation coefficient between them is 0.985 . The maximum value of the reconstructed force is $220 \mathrm{~N}$, leading to an error of less than $10 \%$.

As discussed, the results imply that the impact occurred at location 3, based on the unique shape of a normal impact force. In practice, however, this finding can be questionable, and theoretically without any objective views we can only conclude that the impact occurred at one of two locations (locations 1 and 3).

\subsubsection{Even-determined case}

For an even-determined problem with four inputs (impact locations) and four outputs (measurement points) three different arrangements of measuring points were considered. The measuring points were selected from the left part, the right part, and both parts of the measurement point grid in Figure 5, respectively. 


\subsubsection{Measurement points on left side}

Figure 12 shows the reconstructed impact forces at four potential impact locations incorporating measuring points 3, 5, 9 and 11 located on the left side of the grid. At first glance, based on Figure 12 (b) and (d), impact locations 2 and 4 can be rejected as potential candidates since the magnitude of the reconstructed forces at the two locations is virtually zero.

In Figure 12 (a), the reconstructed impact force at location 1 shows a strange shape with four peaks, in which the first peak is lower than the second. This is unrealistic for a normal impact force, leading to the conclusion that there is a low likelihood of this location being the actual impact location. The shape of the reconstructed force at location 3 (Figure 12 (c)) is smoother and similar to that for an impact force. The correlation coefficients between the reconstructed forces and the actual forces from $t=0 \mathrm{~ms}$ to $t=30 \mathrm{~ms}$ for locations 1 and 3 are 0.591 , and 0.960 , respectively. Moreover, the maximum amplitudes of the reconstructed forces for locations 1 and 3 are $145 \mathrm{~N}$ and $217 \mathrm{~N}$ respectively, with an error of $41 \%$ and $11 \%$, respectively, compared to that of the actual force.

To refine the result, a further approach with only two possible impact locations, locations 1 and 3, and with the previous four measurement points was performed. Depicted in Figure 13, the reconstructed impact force at location 1 has a lower peak than that in Figure 12 (a) and fluctuates abnormally, indicating that this is less likely to be the actual impact location. The maximum amplitudes of the reconstructed forces for locations 1 and 3 are $86 N$ and $210 N$, respectively, showing an error of $65 \%$ and $14 \%$ compared to that of the actual impact force. The correlation coefficient between the reconstructed force and the actual force from $t=0 \mathrm{~ms}$ to $t=30 \mathrm{~ms}$ for location 3 was improved to 0.980 .

\subsubsection{Measurement points on right side}

The reconstructed impact forces at four possible locations using measuring points 4, 6, 10 and 12 on the right side of the measuring point grid are illustrated in Figure 14. From visual assessment, the impact force at location 3 is easily identifiable, as the reconstructed forces at the other locations are less 
likely to be impact forces. The reconstructed forces at locations 1 and 2 do not look like a normal impact force and the amplitude of the first peak at both locations is lower than that of the following peak. Likewise, the shape of the reconstructed force at location 4 is not similar to the normal shape of an impact, though the first peak of the reconstructed force $(\sim 118 N)$ is higher than the following one. Though this finding can be questioned, assuming that no knowledge of the actual force is available, it can be claimed that the likelihood of location 3 being the actual impact location is higher than that of the other three locations. The maximum magnitude of the reconstructed force at location 3 is $181 \mathrm{~N}$, giving an error of $26 \%$ compared to that of the actual force. The correlation coefficient between the reconstructed force at location 3 and the actual force from $t=0 \mathrm{~ms}$ to $t=30 \mathrm{~ms}$ is 0.928 .

\subsubsection{Measurement points on both sides}

The reconstructed impact forces at four potential impact locations through an even-determined problem consisting of evenly distributed measuring points 3, 4, 7 and 8 are shown in Figure 15. As can be seen, locations 2 and 4 can clearly be rejected as impact location candidates, since the reconstructed force is almost zero except for small oscillations. The abnormal shape of the reconstructed force at location 1 leads to a low possibility of this location being the actual impact location. It is nevertheless beneficial to double-check this outcome by retaining this location as a potential candidate.

The reconstructed force at location 3 is very smooth and its maximum magnitude is $248 \mathrm{~N}$, which in fact has an error of less than $2 \%$ compared to that of the actual force. The correlation coefficient between the reconstructed force at location 3 and the actual force from $t=0 \mathrm{~ms}$ to $t=30 \mathrm{~ms}$ is 0.941 .

\section{Automated impact force identification}

In the previous section, successful identification of impact forces was obtained; however, the procedure required manual intervention to qualitatively assess the re-constructed force signals to identify the true impact force based on several qualitative characteristics such as shape and magnitude. Moreover, there could be more than one candidate amongst the reconstructed signals that met the 
requirements of being a true impact force. In those situations Equation 5 therefore needed to be solved again for the remaining candidates which is not efficient nor practical. In this section, a fully automated approach using a quantitative index is presented to identify the true impact location from the reconstructed force signals which eliminates any user intervention and does not require solution of Equation 5 multiple times.

\section{Index-based similarity searching for impact force identification}

The procedure of identifying the true impact force location from a set of reconstructed force signals, introduced in this section, is based on the concept of similarity searching for time-series using wavelet transformation [28]. An indexing strategy is proposed based on the obtained wavelet coefficients for each reconstructed force signal. It is expected that the true impact force results in the highest index.

Continuous wavelet transform (CWT) is a mathematical transformation to measure the similarity between a signal and an analysing function $\psi(t)$ as $[29,30]$,

$$
W(a, b)=\frac{1}{\sqrt{a}} \int f(t) \psi\left(\frac{t-b}{a}\right) d t
$$

where $a$ and $b$ are dilation (scale) and translation parameters, respectively . Both are real numbers and $a$ must be positive. $W$ is the wavelet coefficient and $f(t)$ is the analysed signal. Wavelet coefficient is a measure of similarity between the mother wavelet and the signal itself at a given scale and translation parameter. In other words, CWT coefficients refer to the closeness of the signal to the wavelet. Large values of wavelet coefficients represent better correlation between the signal and the wavelet at that particular location and scale value. If the signal has a major component of the frequency corresponding to the current scale, then the wavelet at the current scale will be similar or close to the signal at that particular location where this frequency component exists. This characteristic of wavelet transform allows identifying the time localised patterns within a signal. In the context of force identification, if the mother wavelet has similar shape to a true impact force, then, it is expected that the obtained 
wavelet coefficients for true impact force to be higher than the counterparts for the false reconstructed signals. Thus, a similarity index is proposed as,

$$
\delta=\sum_{a} \sum_{b}|W(a, b)|
$$

where $\delta$ is the force identification index and it is expected to have the maximum value of $\delta$ corresponding to the true impact force. In this study, a mother wavelet from Daubechies family (Daub12) is selected as it is similar to an impact force signal.

The proposed procedure was conducted to identify the true impact force for the reconstructed impact forces shown in Figures 10, 12, 14 and 15 and the results are presented in Figures 16 (a-d), 17(a-d), 18(a-d) and 19(a-d), respectively. For each potential impact location, the wavelet coefficients were first obtained for a wide range of scale and translation parameters. The scale range is up to 1000 samples and the translation parameter range is up to 750 samples covering the entire reconstructed force history window. It should be noted that in these figures the reconstructed force is plotted against 'sample'. The heatmap of the wavelet coefficients illustrates the intensity of the coefficients; the lighter the colour, the higher the coefficient. As seen, in all the cases, the lightest colour in the contours corresponds to parts of the signal where there are localised half-sine shape features in the signal. Based on the obtained coefficients, the proposed index is calculated for each potential impact location in the four investigated cases and the results are shown in bar plots in Figures 16-19 (e). As illustrated, promising identification of the true impact force is obtained for all four investigated cases as the maximum of index corresponds to the location 3 which is the true impact force.

\section{Concluding remarks}

Identification of an impact force exerted on a lattice truss core composite sandwich panel was investigated through one-to-one and superposition approaches. The one-to-one approach reconstructing the impact forces independently for all possible locations was shown to fail to detect the true impact 
location. In the superposition approach, the impact force magnitude and location were concurrently inversely determined through an extended matrix form of the convolution equation based on the velocity signals captured at the multiple points. It was supposed that an impact force had been incurred at one of four likely locations. Two scenarios with different numbers of measurement points (12 and 4) leading to over-determined and even-determined problems were investigated. Based on assessment of the reconstructed forces, the impact location was identifiable as location 3, which showed the typical normal shape of an impact force.

It was demonstrated that too many measurement points, as in the over-determined problem, did not necessarily enhance the efficiency and accuracy of the proposed method, as the results of an evendetermined problem showed errors of less than $2 \%$ with an arrangement of measuring points. It was also found that different arrangements of measuring points all led to identification of the location and magnitude of the impact force. However, the accuracy of the reconstructed force using an evenly distributed arrangement of the measurement points on both left and right sides was higher, producing the minimum error for the maximum amplitude of the reconstructed force.

Furthermore, since it was necessary to apply the algorithm twice to maximise the confidence of identifying the impact location, a quantitative index based on the concept of similarity search for timeseries using wavelet transformation was proposed and it was demonstrated that the index can successfully identify the true impact force in a fully automated way. This is a significant finding since it minimises the need for human interaction and therefore yields a more robust, industrial applicable technique. 


\section{Reference}

1. Wang, B., et al., Mechanical behavior of the sandwich structures with carbon fiber-reinforced pyramidal lattice truss core. Materials \& Design, 2010. 31(5): p. 2659-2663.

2. Liu, J., L. Xiang, and T. Kan, The effect of temperature on the bending properties and failure mechanism of composite truss core sandwich structures. Composites Part A: Applied Science and Manufacturing, 2015. 79: p. 146-154.

3. Liu, J., et al., Effects of thermal exposure on mechanical behavior of carbon fiber composite pyramidal truss core sandwich panel. Composites Part B: Engineering, 2014. 60: p. 82-90.

4. Wadley, H.N., Cellular metals manufacturing. Advanced Engineering Materials, 2002. 4(10): p. 726-733.

5. $\quad$ Fotouhi, M., et al., Investigation of the damage mechanisms for mode I delamination growth in foam core sandwich composites using acoustic emission. Structural Health Monitoring, 2015. 14(3): p. 265-280.

6. Zhang, M., et al., Hybrid reconstruction method for indirect monitoring of an ice load of a steel gate in a cold region. Cold Regions Science and Technology, 2019. 162: p. 19-34.

7. Zhang, M., et al., Indirect monitoring of distributed ice loads on a steel gate in a cold region. Cold Regions Science and Technology, 2018. 151: p. 267-287.

8. Jacquelin, E., A. Bennani, and P. Hamelin, Force reconstruction: analysis and regularization of a deconvolution problem. Journal of Sound and Vibration, 2003. 265(1): p. 81-107.

9. Hansen, P.C., Regularization tools: A Matlab package for analysis and solution of discrete illposed problems. Numerical algorithms, 1994. 6(1): p. 1-35.

10. Hansen, P.C., Rank-deficient and discrete ill-posed problems: numerical aspects of linear inversion. Vol. 4. 1998: Siam.

11. Kundu, T., S. Das, and K.V. Jata, Point of impact prediction in isotropic and anisotropic plates from the acoustic emission data. The Journal of the Acoustical Society of America, 2007. 122(4): p. 2057-2066.

12. Coverley, P. and W. Staszewski, Impact damage location in composite structures using optimized sensor triangulation procedure. Smart materials and structures, 2003. 12(5): p. 795803.

13. Hajzargerbashi, T., T. Kundu, and S. Bland, An improved algorithm for detecting point of impact in anisotropic inhomogeneous plates. Ultrasonics, 2011. 51(3): p. 317-324.

14. Choi, K. and F.-K. Chang, Identification of impact force and location using distributed sensors. AIAA journal, 1996. 34(1): p. 136-142.

15. $\mathrm{Hu}, \mathrm{N}$., et al., An efficient approach for identifying impact force using embedded piezoelectric sensors. International journal of impact engineering, 2007. 34(7): p. 1258-1271.

16. Kalhori, H., M.M. Alamdari, and L. Ye, Automated algorithm for impact force identification using cosine similarity searching. Measurement, 2018. 122: p. 648-657.

17. Dimarogonas, A., Vibration for Engineers. 1996, Prentice-Hall International Editions.

18. Meirovitch, L., Elements of vibration analysis. 1986: McGraw-Hill

19. Inoue, H., J.J. Harrigan, and S.R. Reid, Review of inverse analysis for indirect measurement of impact force. Applied Mechanics Reviews, 2001. 54(6): p. 503-524.

20. Lu, S. and S.V. Pereverzev, Regularization theory for ill-posed problems: selected topics. Vol. 58. 2013: Walter de Gruyter.

21. Yin, S., et al., Pyramidal lattice sandwich structures with hollow composite trusses. Composite Structures, 2011. 93(12): p. 3104-3111.

22. Li, B., et al., Damage localization in composite lattice truss core sandwich structures based on vibration characteristics. Composite Structures, 2015. 126: p. 34-51. 
23. Rothberg, S., et al., An international review of laser Doppler vibrometry: Making light work of vibration measurement. Optics and Lasers in Engineering, 2017. 99: p. 11-22.

24. Kalhori, H., et al., Reconstruction and Analysis of Impact Forces on a Steel-Beam-Reinforced Concrete Deck. Experimental Mechanics, 2016. 56(9): p. 1547-1558.

25. Kalhori, H., L. Ye, and S. Mustapha, Inverse estimation of impact force on a composite panel using a single piezoelectric sensor. Journal of Intelligent Material Systems and Structures, 2017. 28(6): p. 799-810.

26. Kalhori, H., et al. Identification of Location and Magnitude of Impact Force on a Composite Sandwich Structure With Lattice Truss Core. in ASME 2015 International Mechanical Engineering Congress and Exposition. 2015. American Society of Mechanical Engineers.

27. Kalhori, H., et al., Impact force reconstruction on a concrete deck using a deconvolution approach. 8th Australasian Congress on Applied Mechanics: ACAM 8. Barton, ACT: Engineers Australia, 2014: p. 763-771.

28. Popivanov, I. and R.J. Miller. Similarity search over time-series data using wavelets. in icde. 2002. IEEE.

29. Kalhori, H., Inverse Algorithm for Impact Force Identification on Engineering Structures. PhD dissertation, The University of Sydney, 2017.

30. Kalhori, H., B. Halkon, and M. Alamdari. Wavelet transform-based strategy for identifying impact force on a composite panel. in Proceedings of the 26th International Congress on Sound and Vibration, ICSV 2019. 2019. 
Table 1. Material parameters of a single lamina and a truss

\begin{tabular}{ccccccc}
\hline & $\boldsymbol{E}_{\mathbf{1}}(\mathbf{G P a})$ & $\boldsymbol{E}_{\mathbf{2}}(\mathbf{G P a})$ & $\boldsymbol{G}_{\mathbf{1 2}}(\mathbf{G P a})$ & $\boldsymbol{G}_{\mathbf{2 3}}(\mathbf{G P a})$ & $\boldsymbol{v 1 2}_{2}$ & $\boldsymbol{v 2 3}$ \\
\hline Lamina & 123.0 & 8.3 & 4.8 & 3.9 & 0.30 & 0.38 \\
\hline Bar & 60.0 & & & & 0.30 & \\
\hline
\end{tabular}

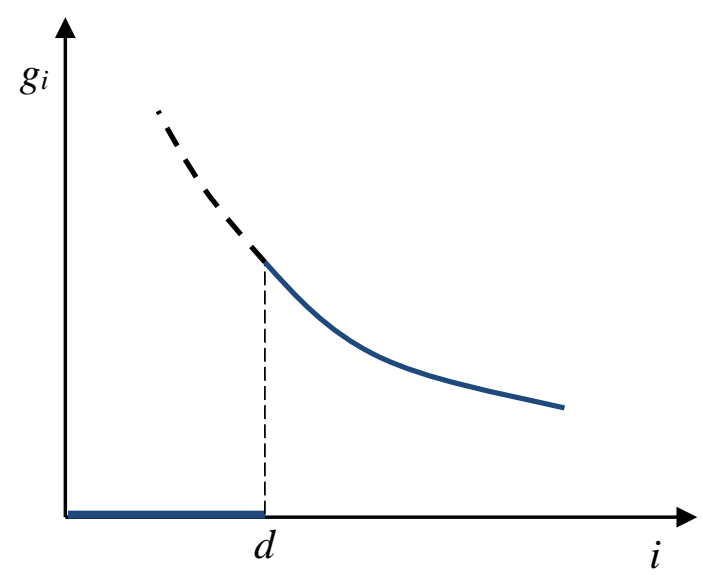

Figure 1. Diagonal elements of matrix $S_{d}^{\ell}$ in spectral cut-off regularisation method. 


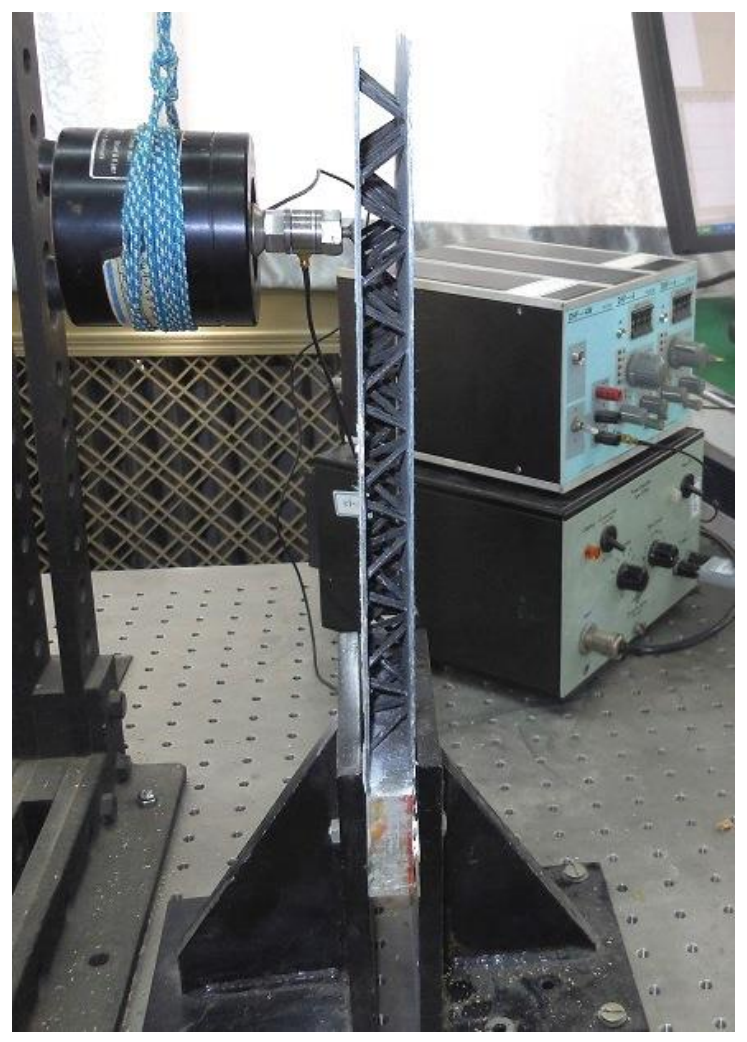

(a)

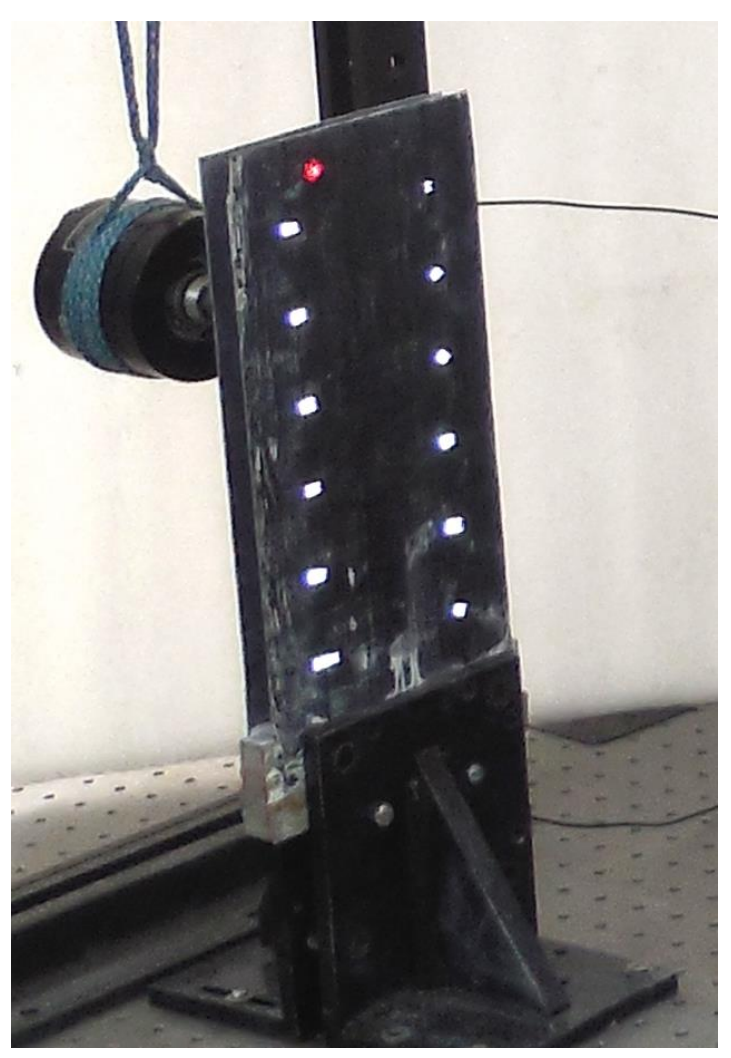

(b)

Figure 2. A lattice truss core composite sandwich panel; (a) side view, and (b) measurement points with patches of reflective tape 


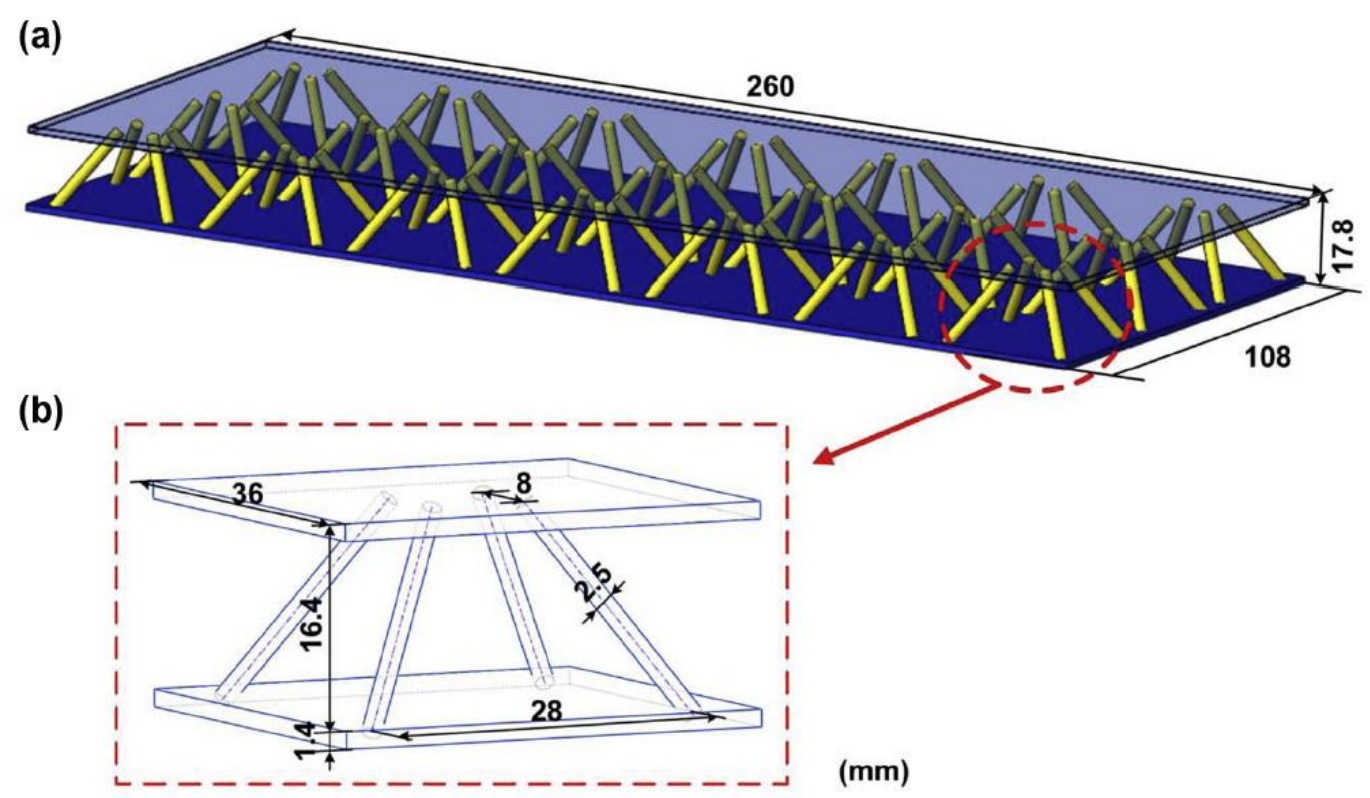

Figure 3. Schematic of (a) the specimen, and (b) the unit cell [22].

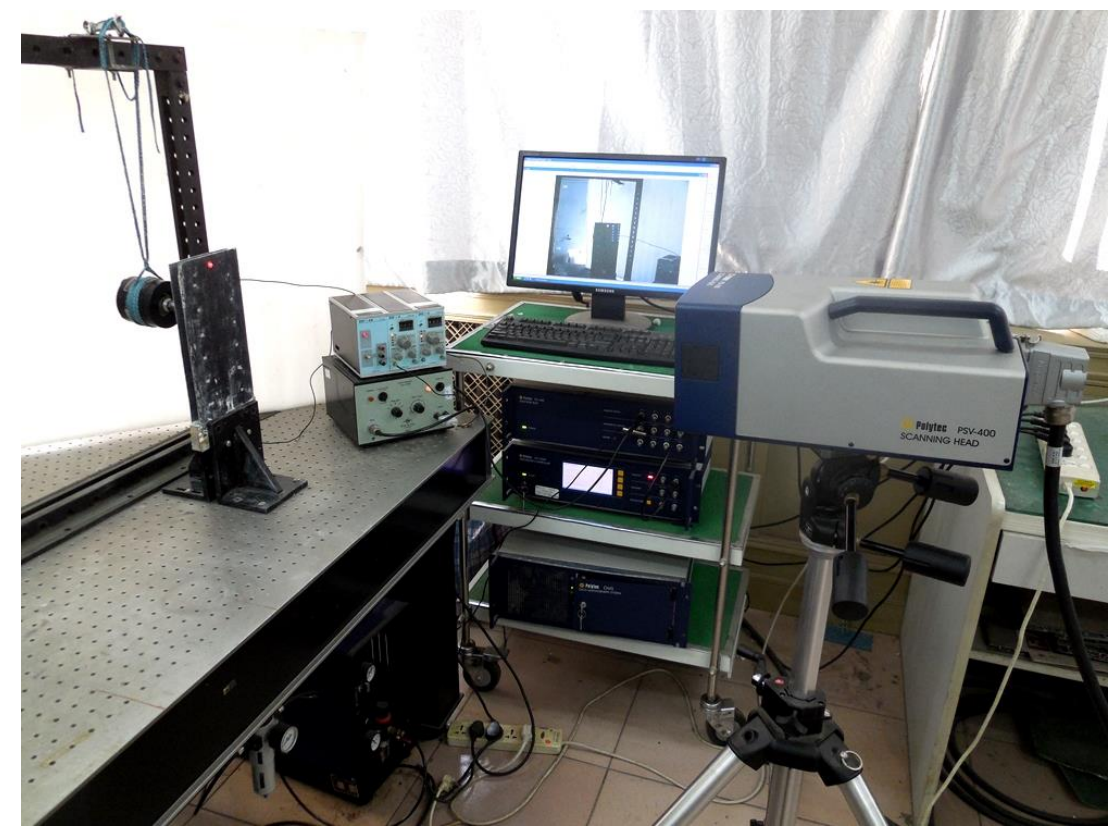

Figure 4. Experimental set-up including the SLDV, shaker, power amplifier and data acquisition system. 


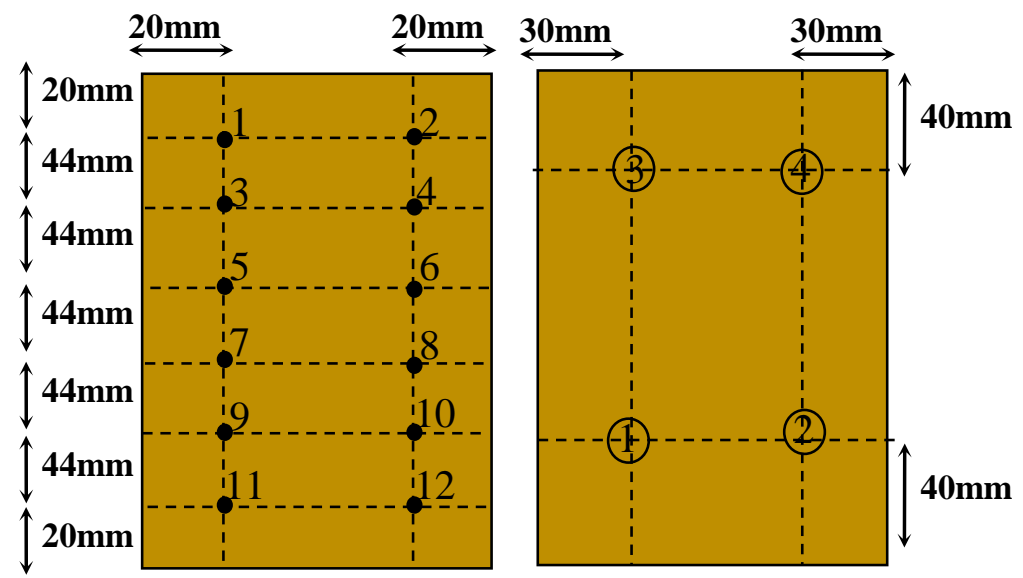

Figure 5. Measurement points at front (left); Impact locations at back (right). 


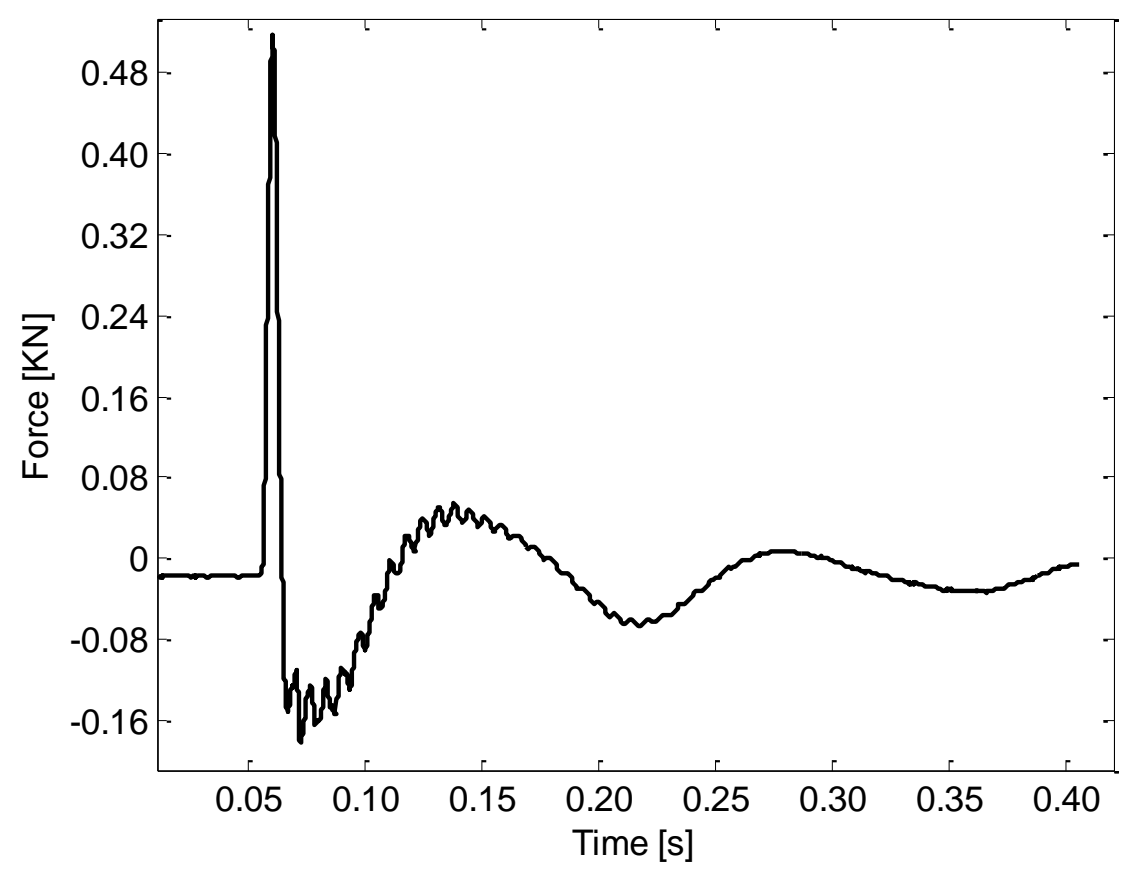

Figure 6. Actual force applied by the shaker at location 2 .

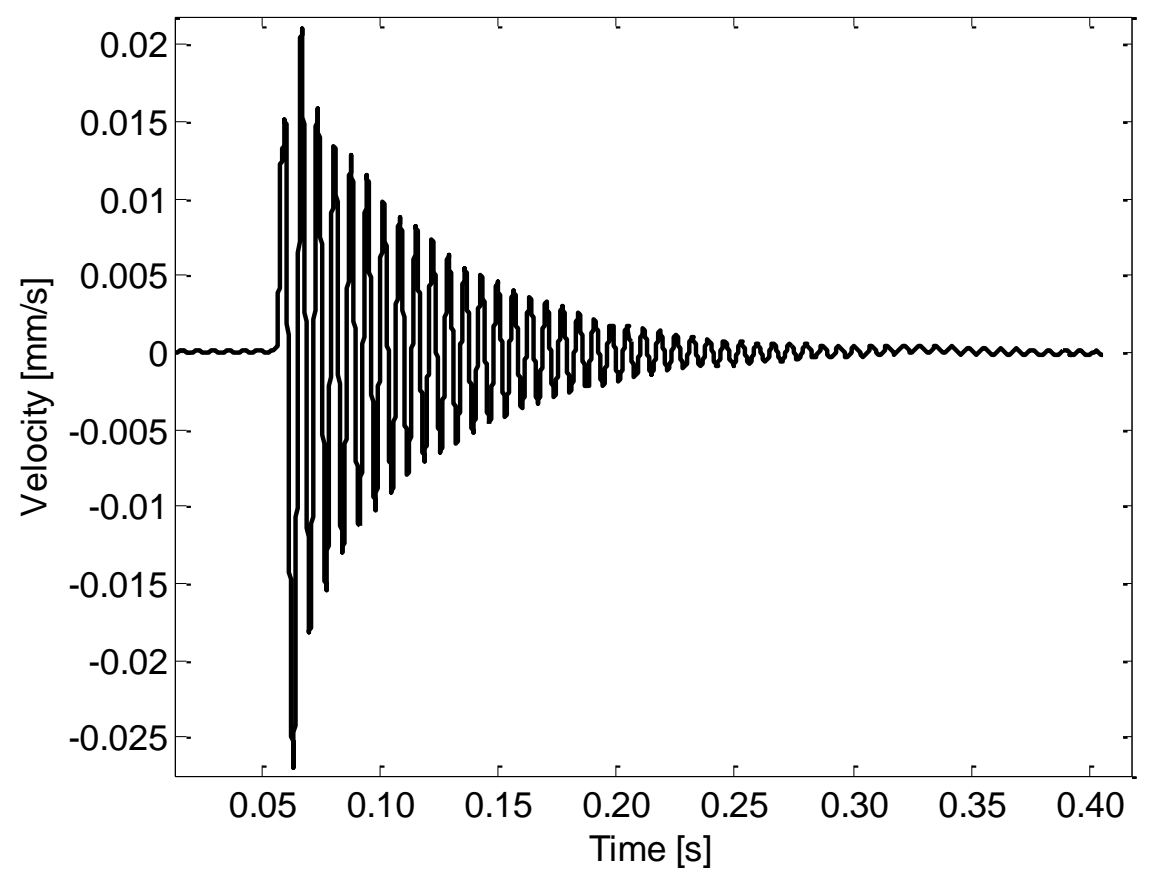

Figure 7. Velocity response captured at point 3. 
(a)

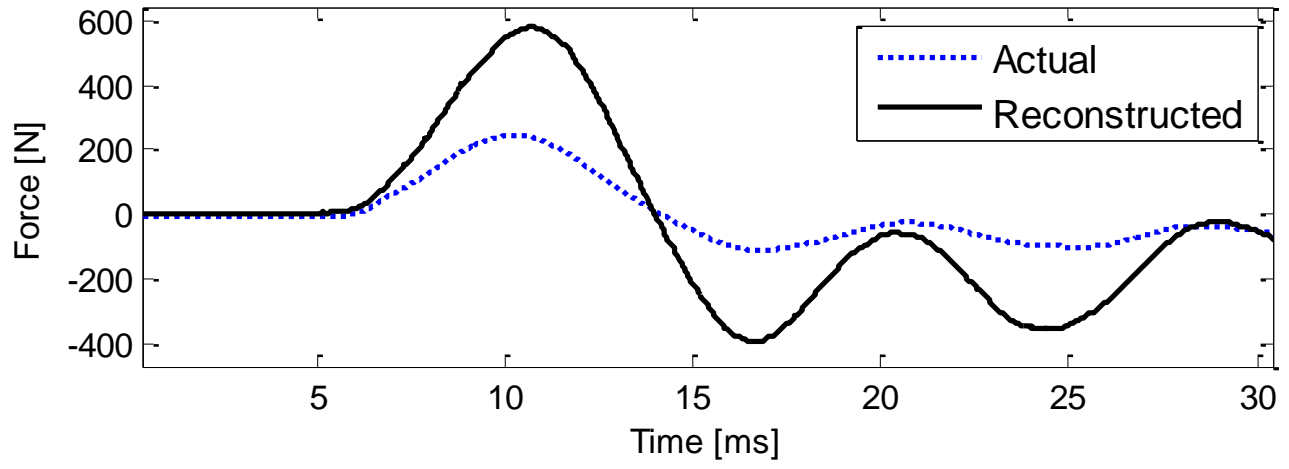

(b)

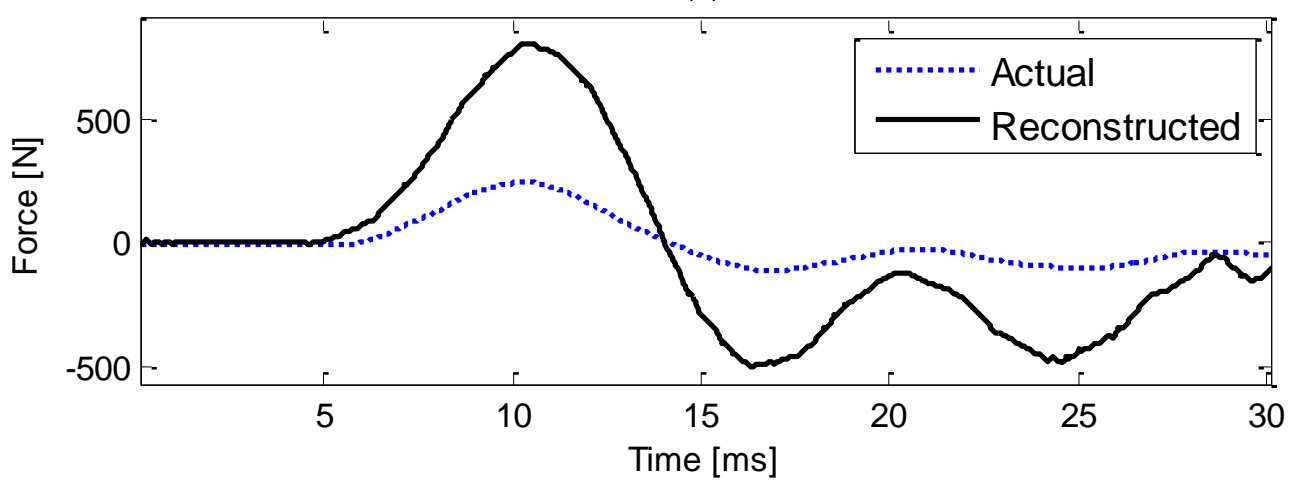

(c)

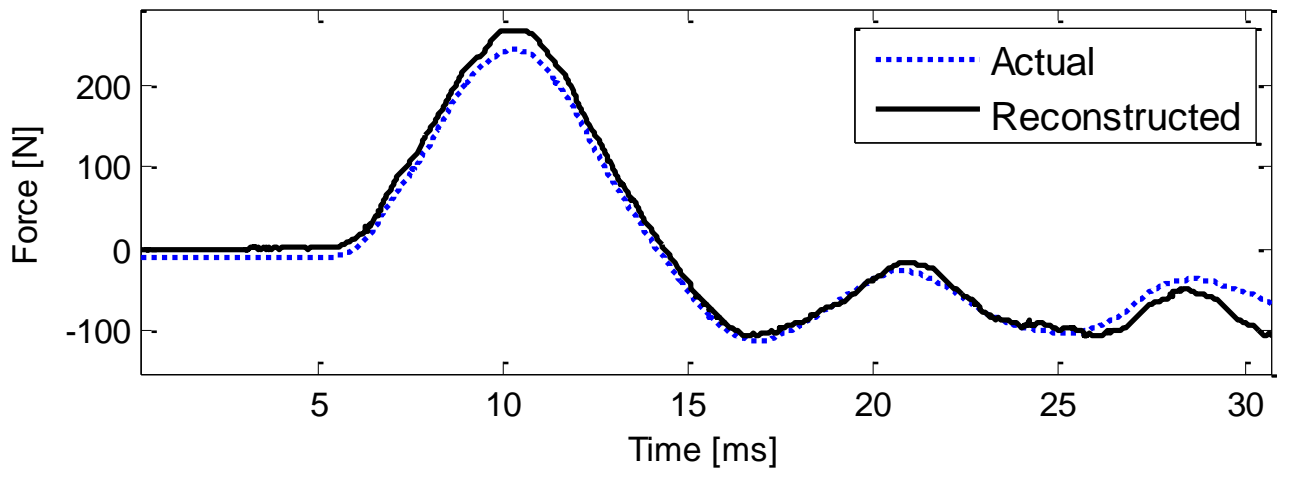

(d)

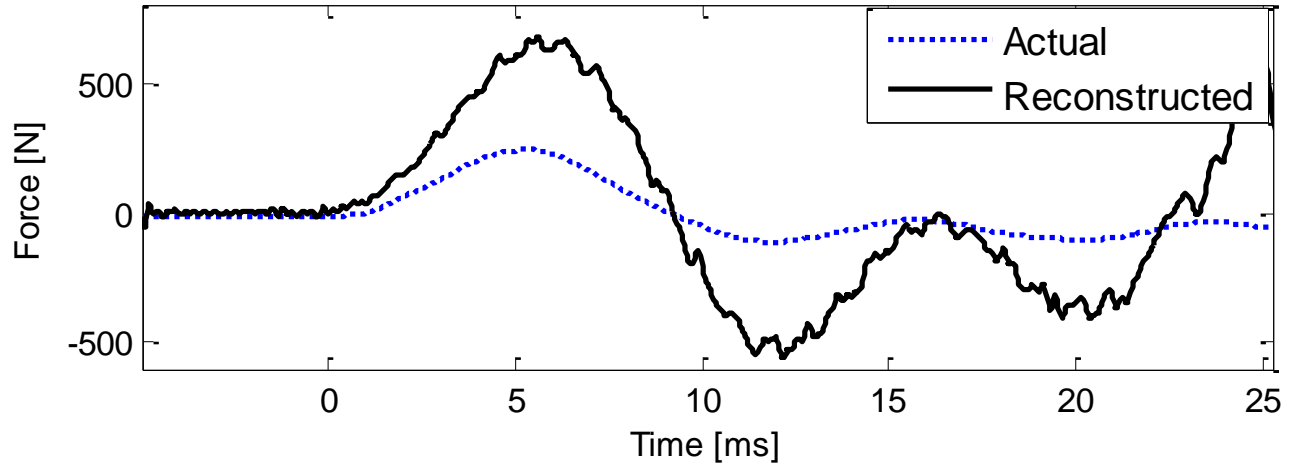

Figure 8. Reconstructed forces through one-to-one approach for locations (a) 1, (b) 2, (c) 3 and (d) 4. 


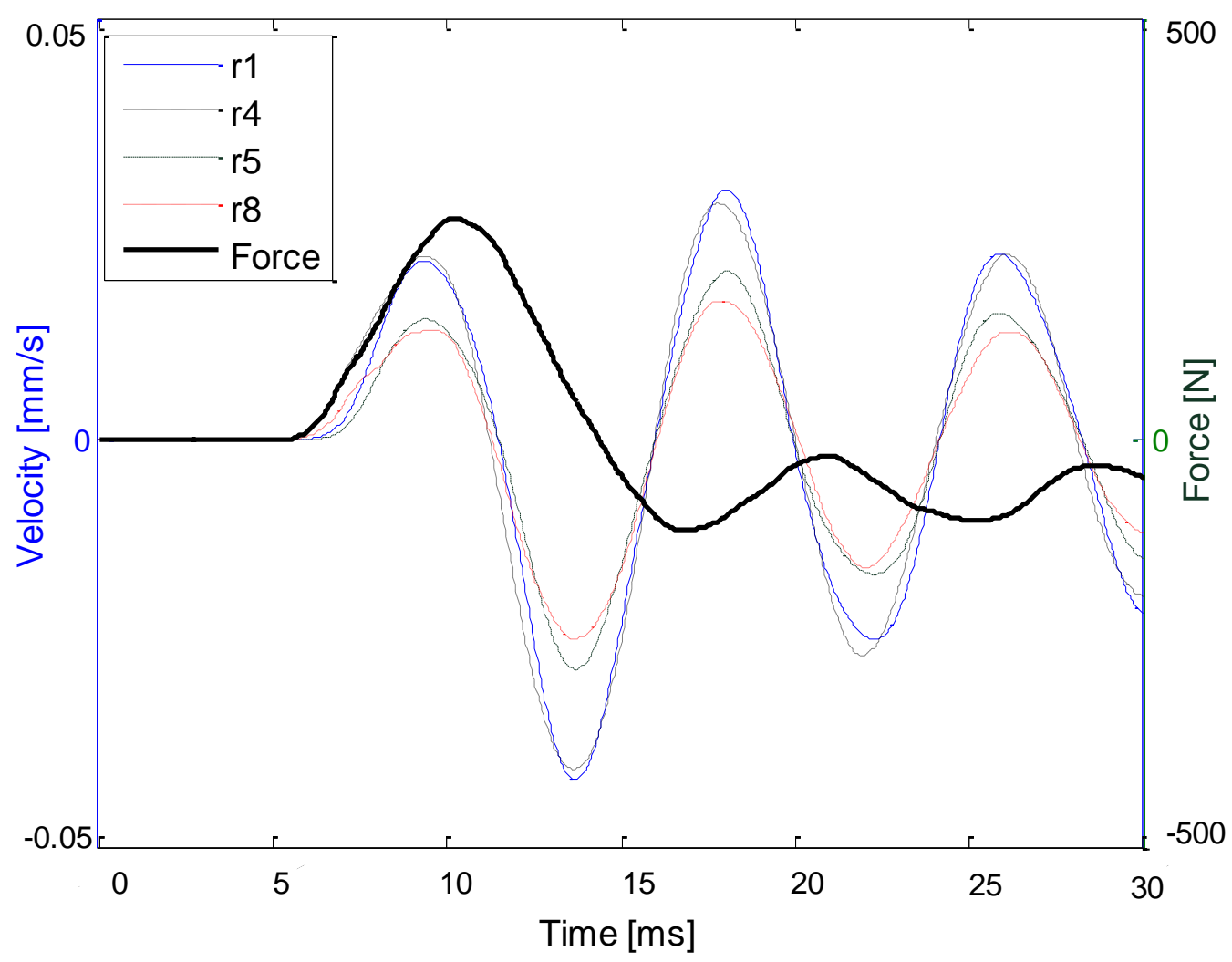

Figure 9. Actual force applied at location 3 and corresponding measurements of velocity at points 1, 4, 5 and 8 up to $30 \mathrm{~ms}$. 
(a)

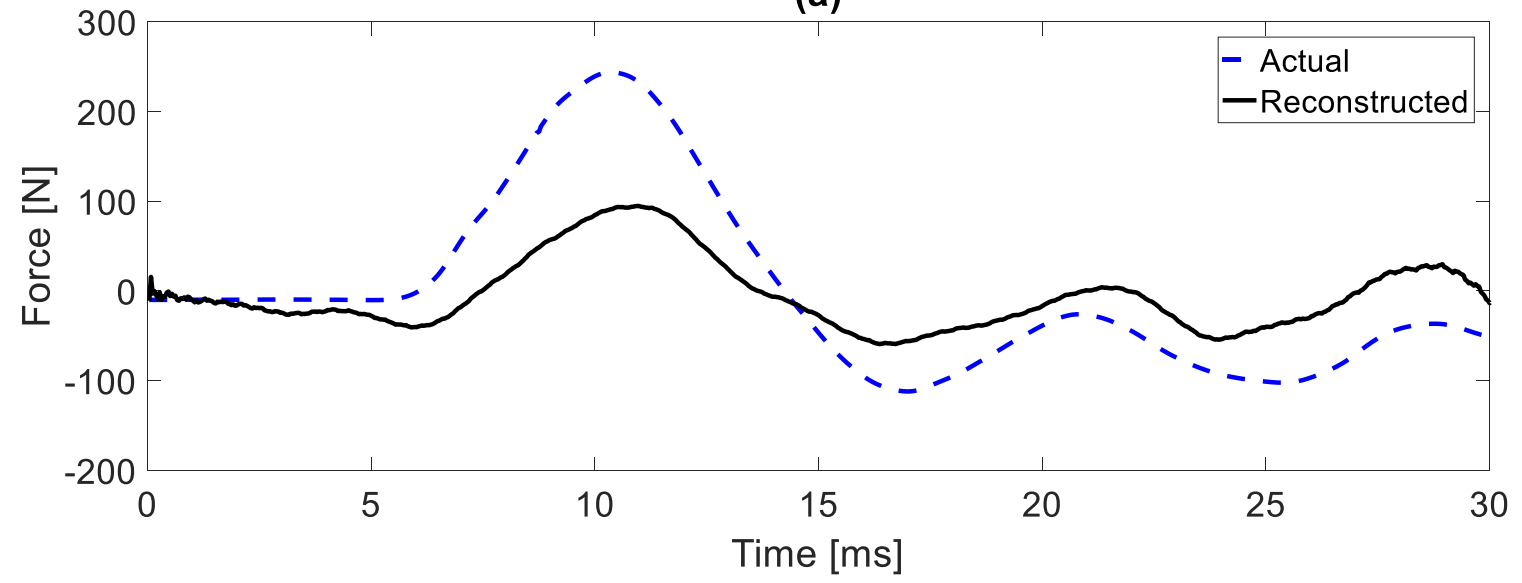

(b)

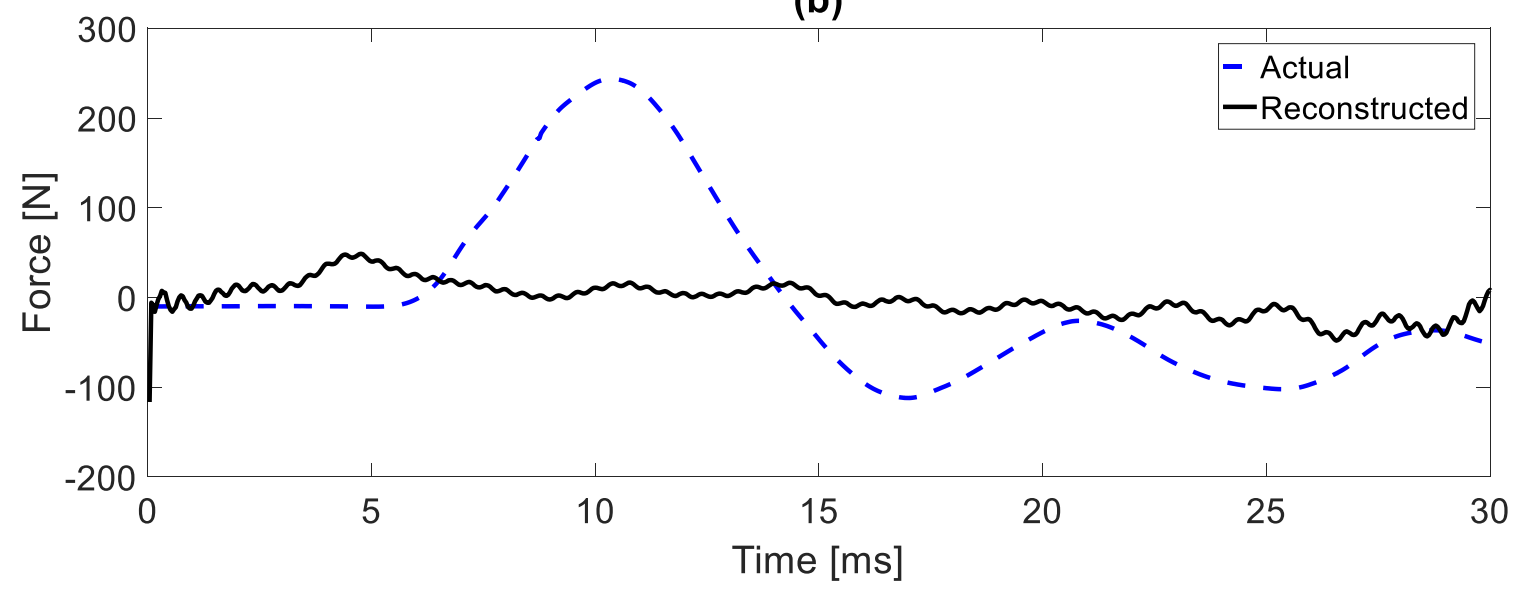

(c)

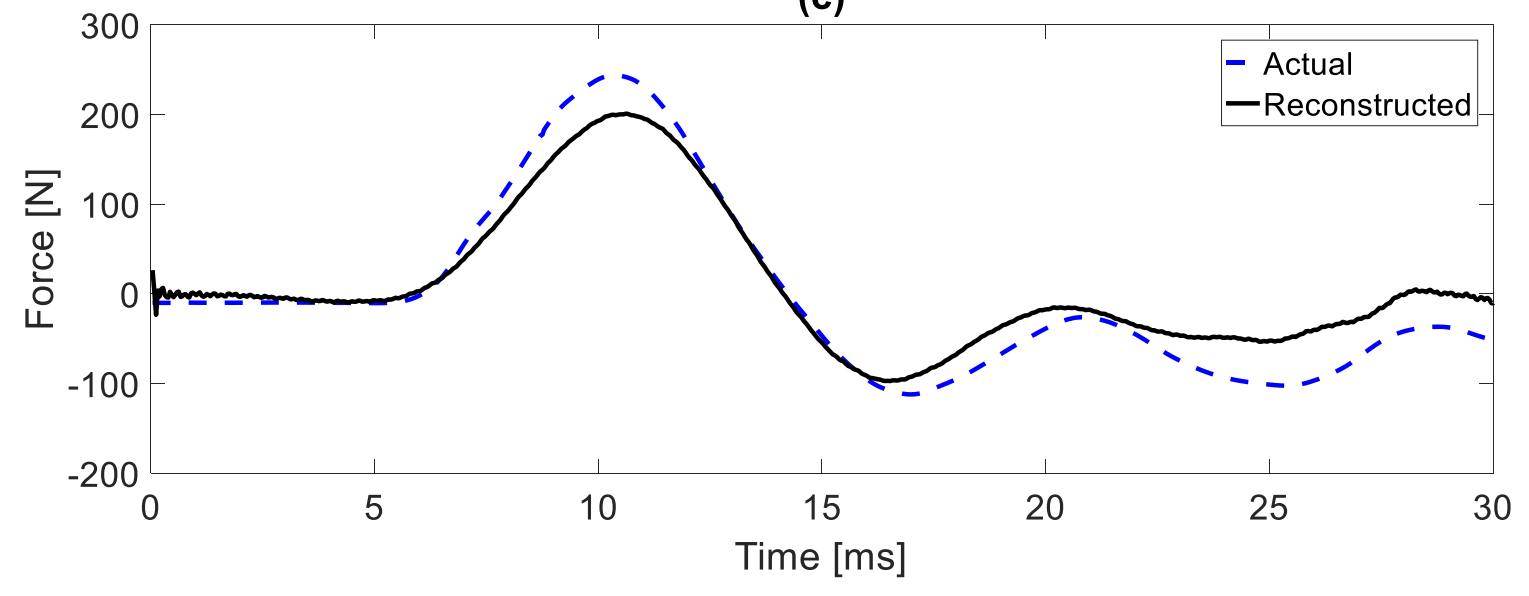


(d)

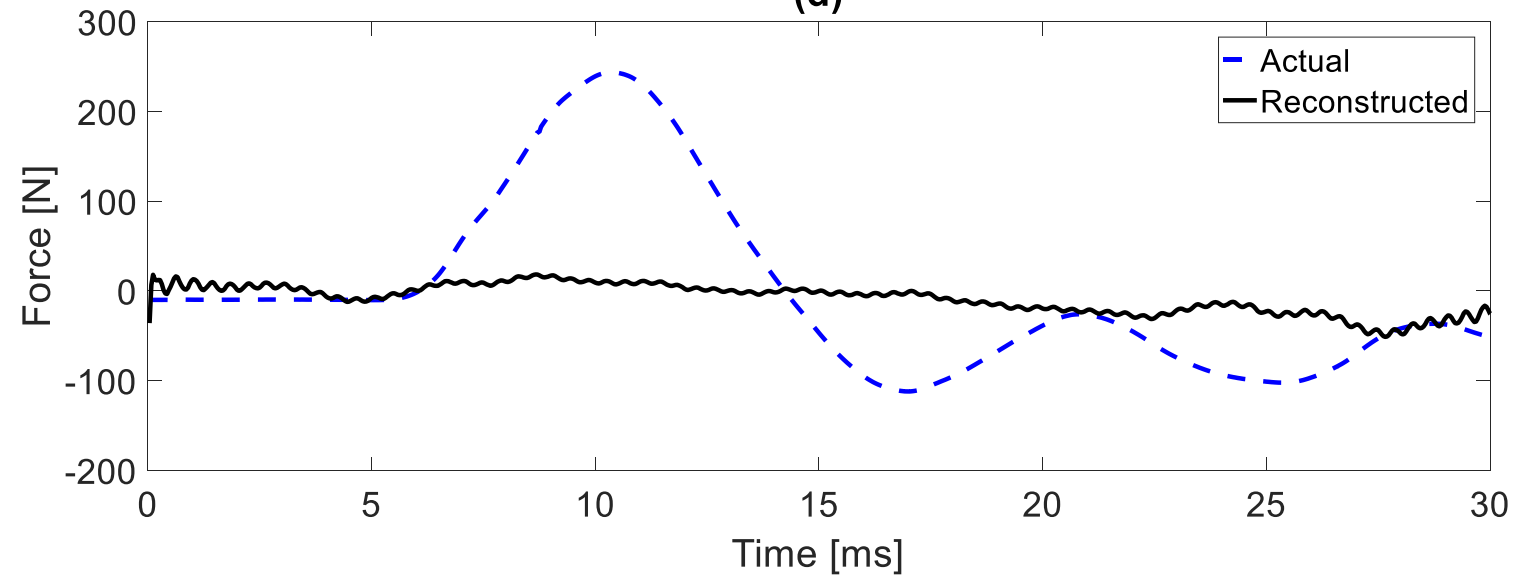

Figure 10. Reconstructed impact forces at (a) location 1, (b) location 2, (c) location 3 and (d) location 4 using velocity responses measured at 12 points.

(a)

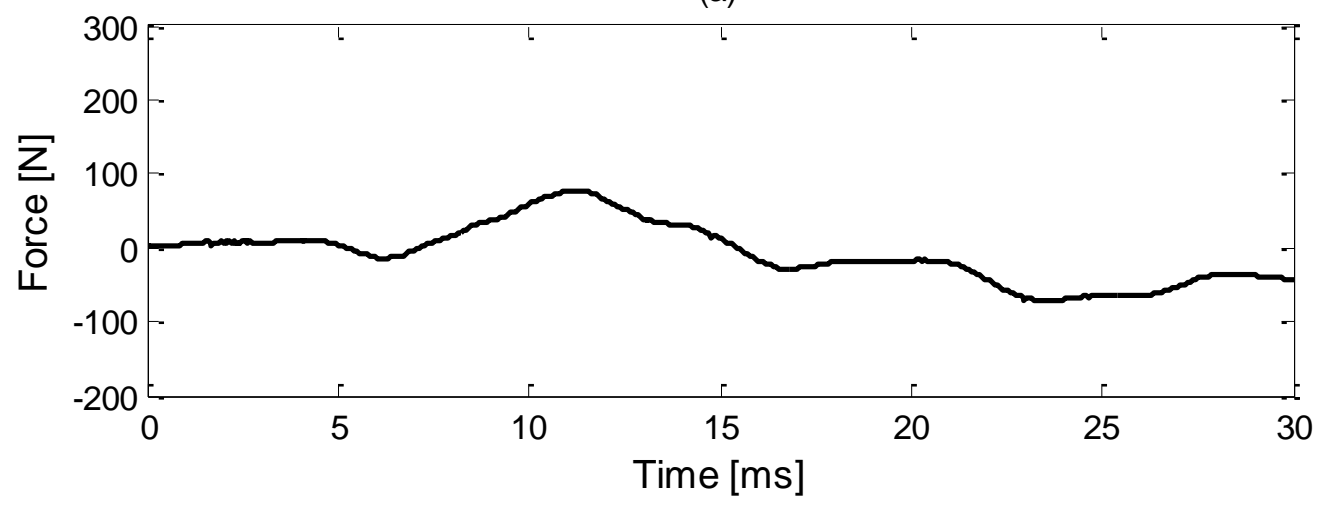

(b)

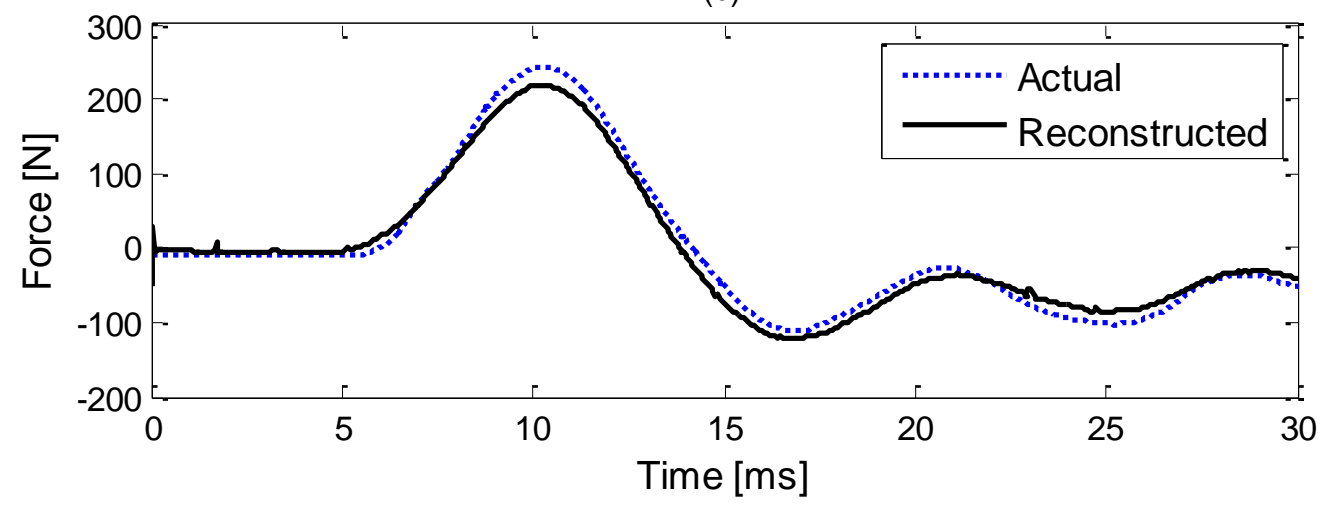

Figure 11. Reconstructed impact forces at (a) location 1 and (b) location 3 through an over-determined problem consisting of 2 impact locations and 12 measuring points. 
(a)

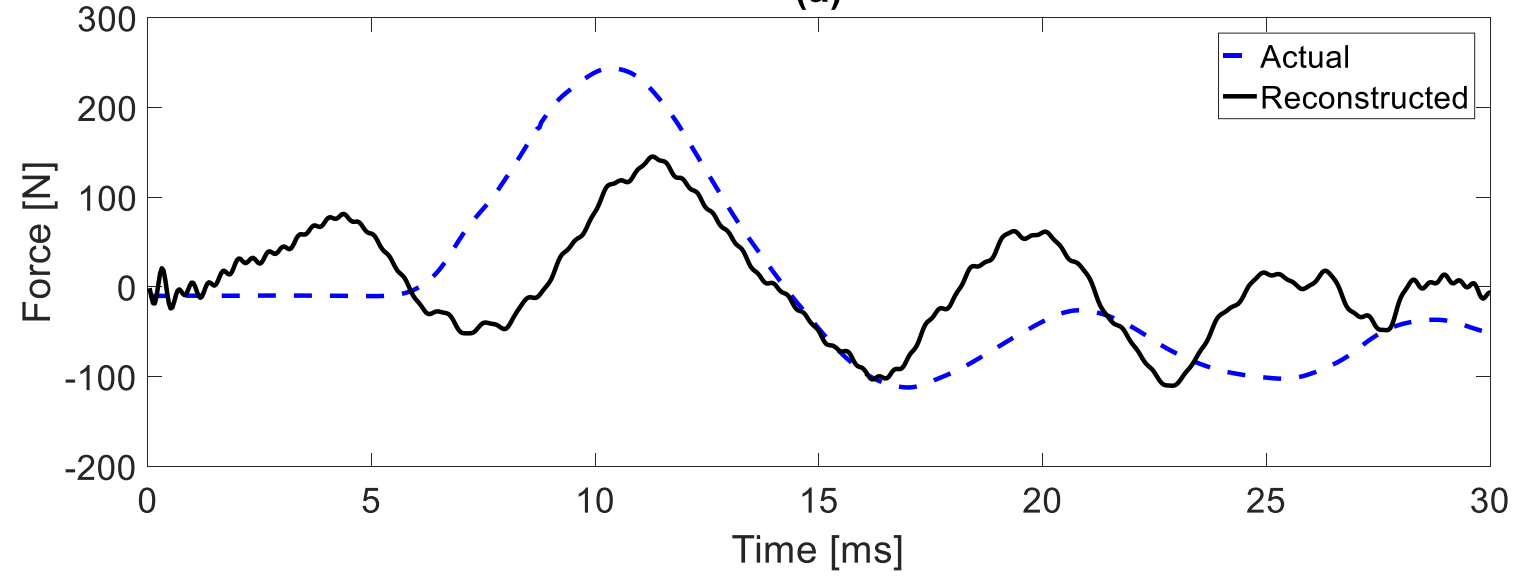

(b)

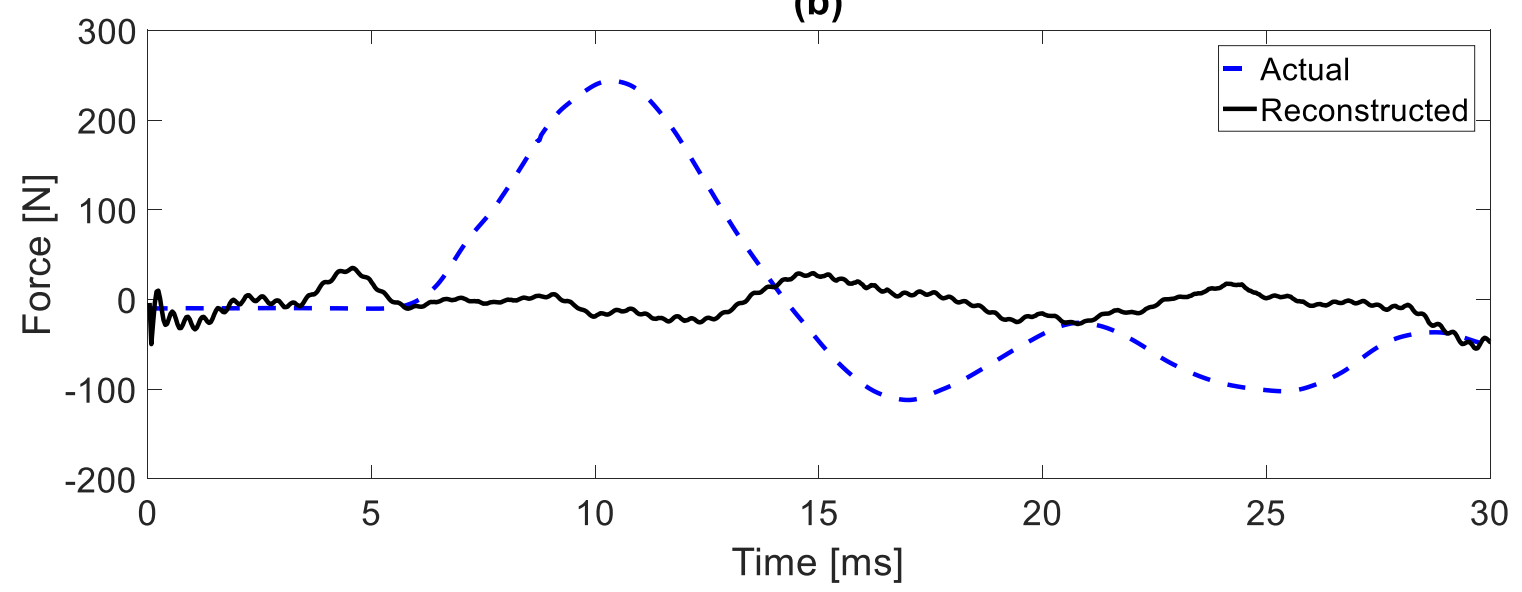

(c)

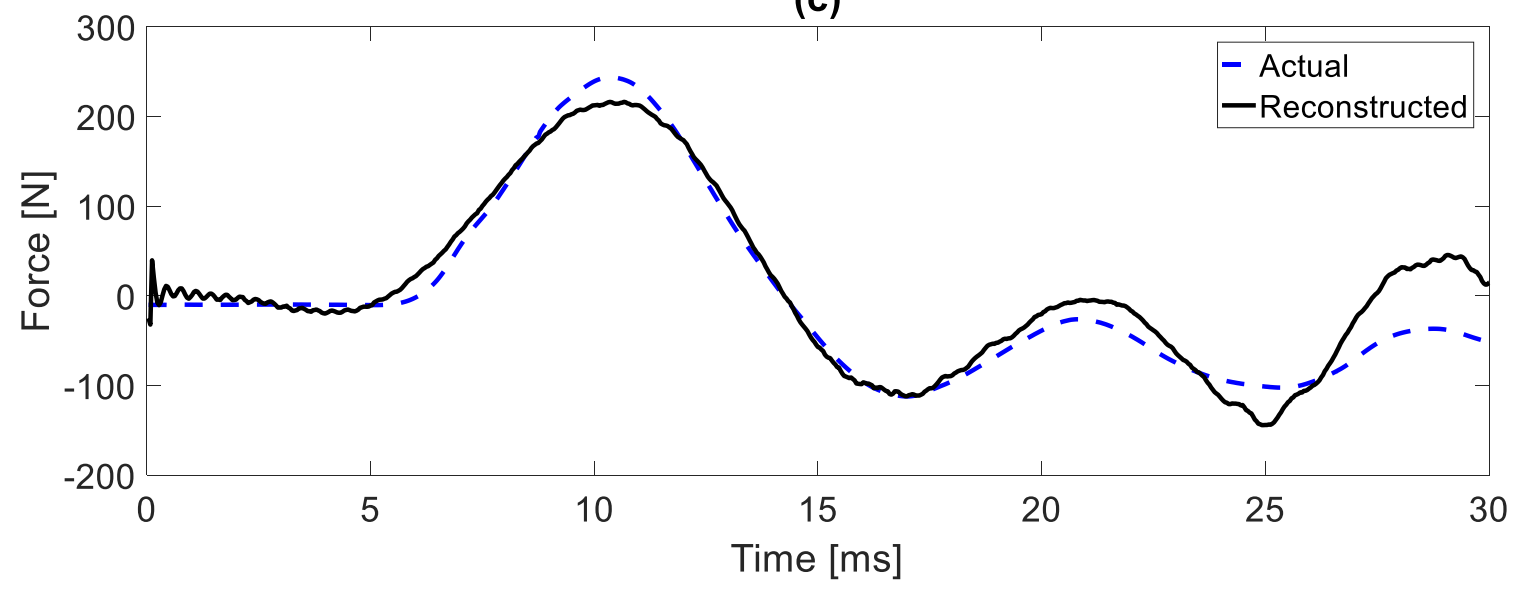


(d)

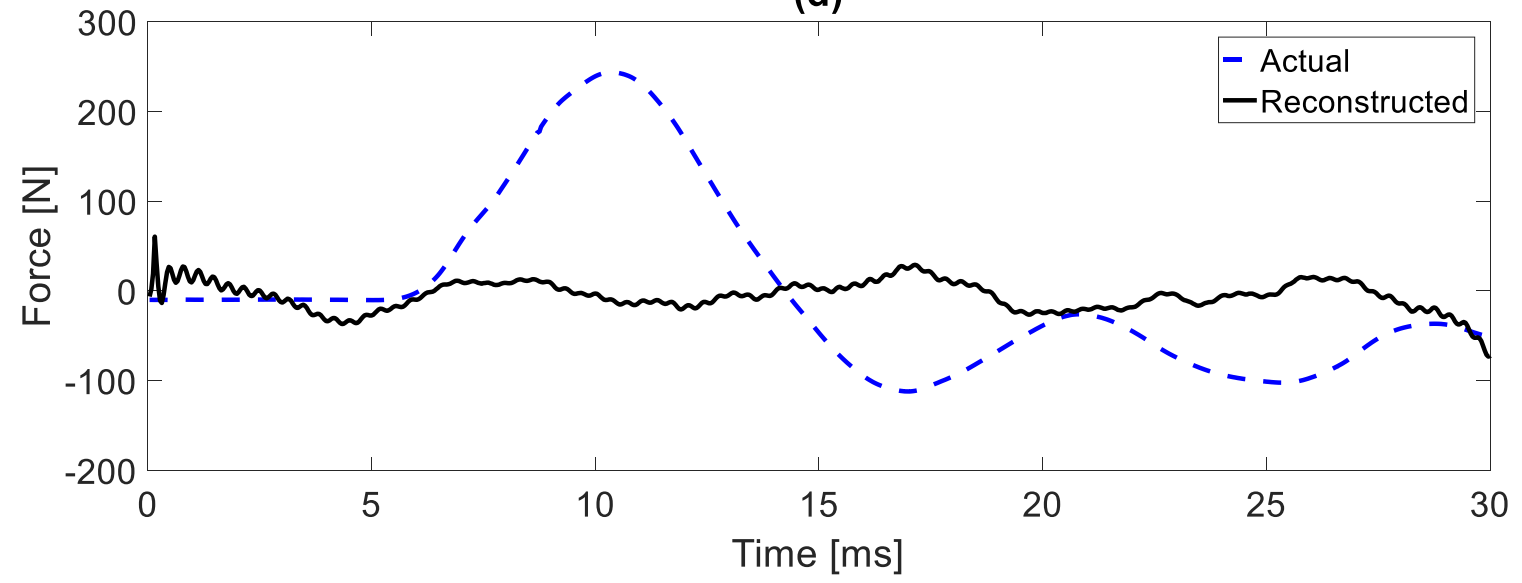

Figure 12. Reconstructed impact forces at (a) location 1, (b) location 2, (c) location 3 and (d) location 4 using velocity responses measured at points 3, 5, 9 and 11 .

(a)

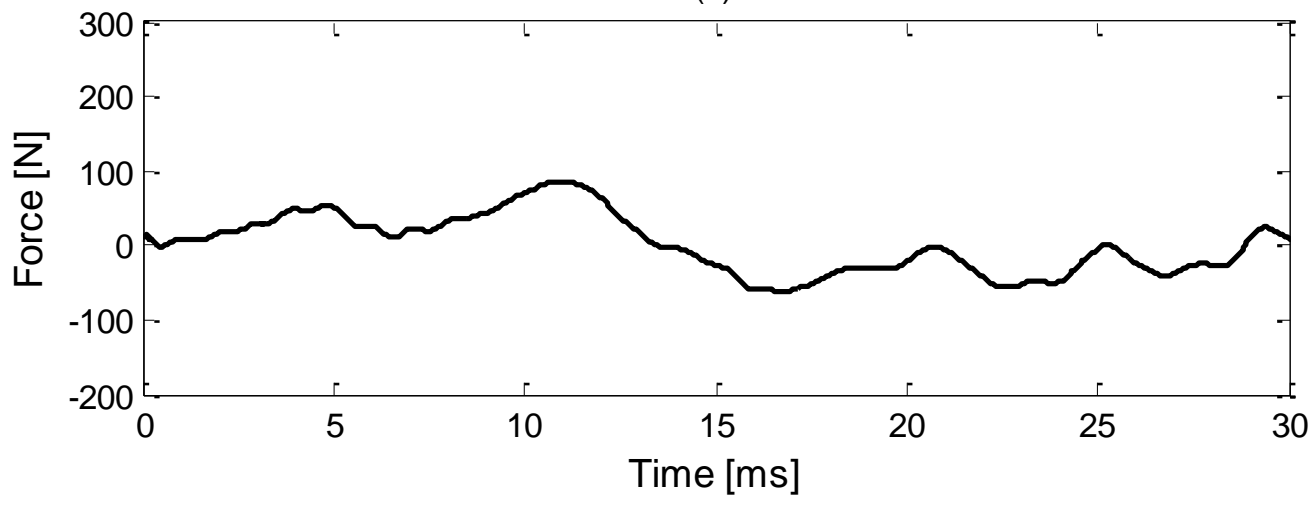

(b)

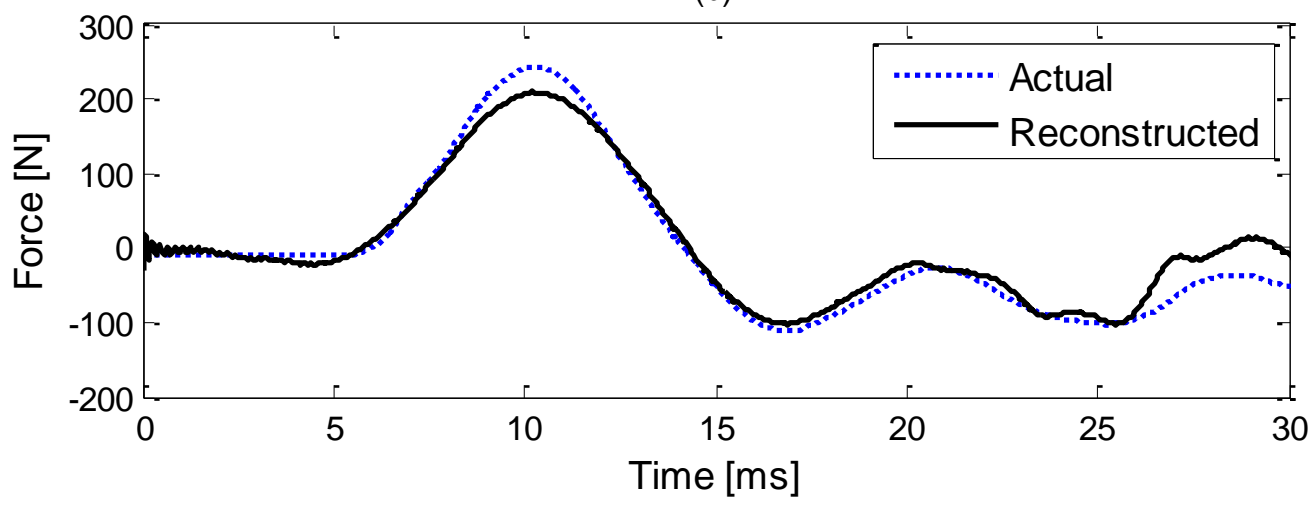

Figure 13. Reconstructed impact forces at (a) location 1 and (b) location 3 through an over-determined problem using impact locations 1 and 3 and measuring points 3, 5, 9 and 11 . 
(a)

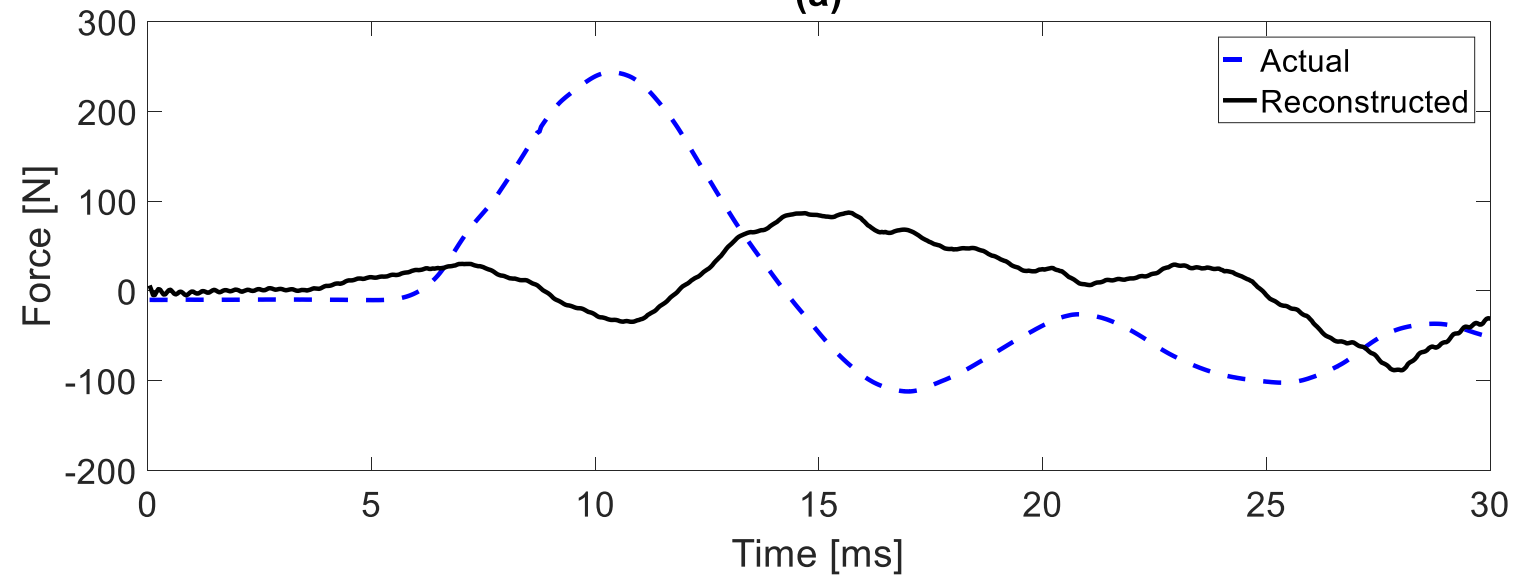

(b)

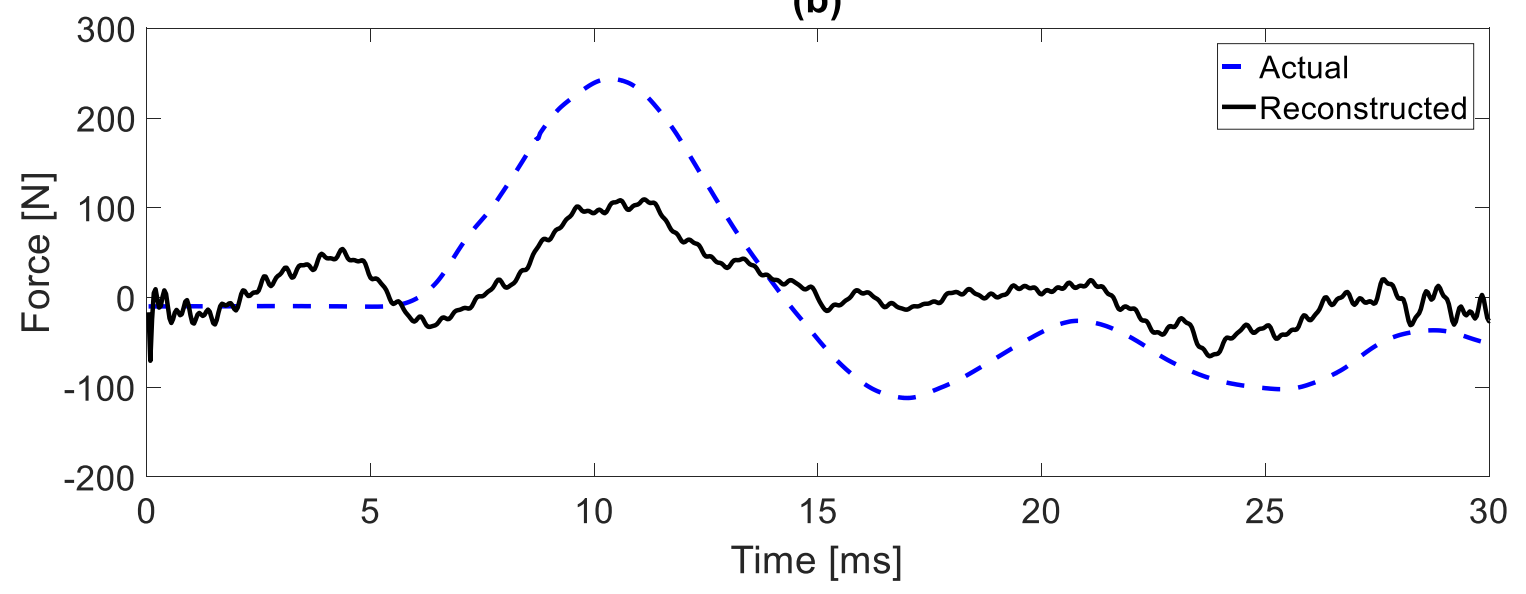

(c)

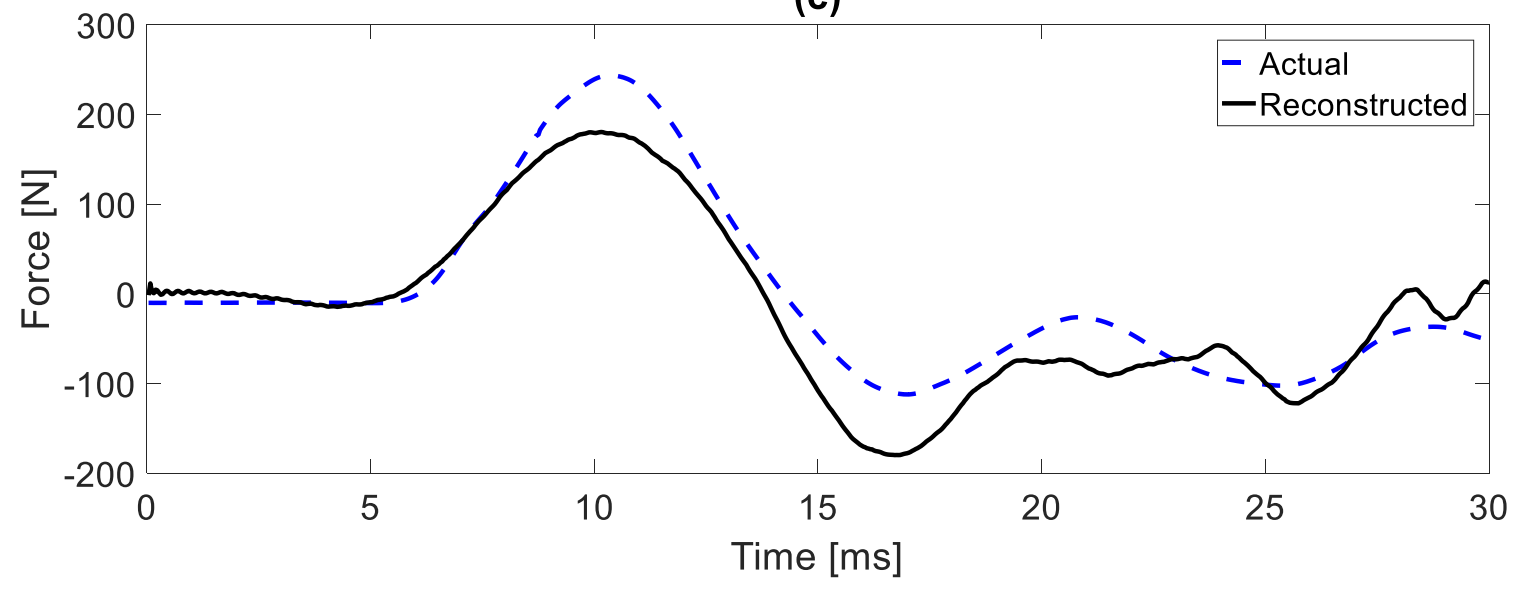


(d)

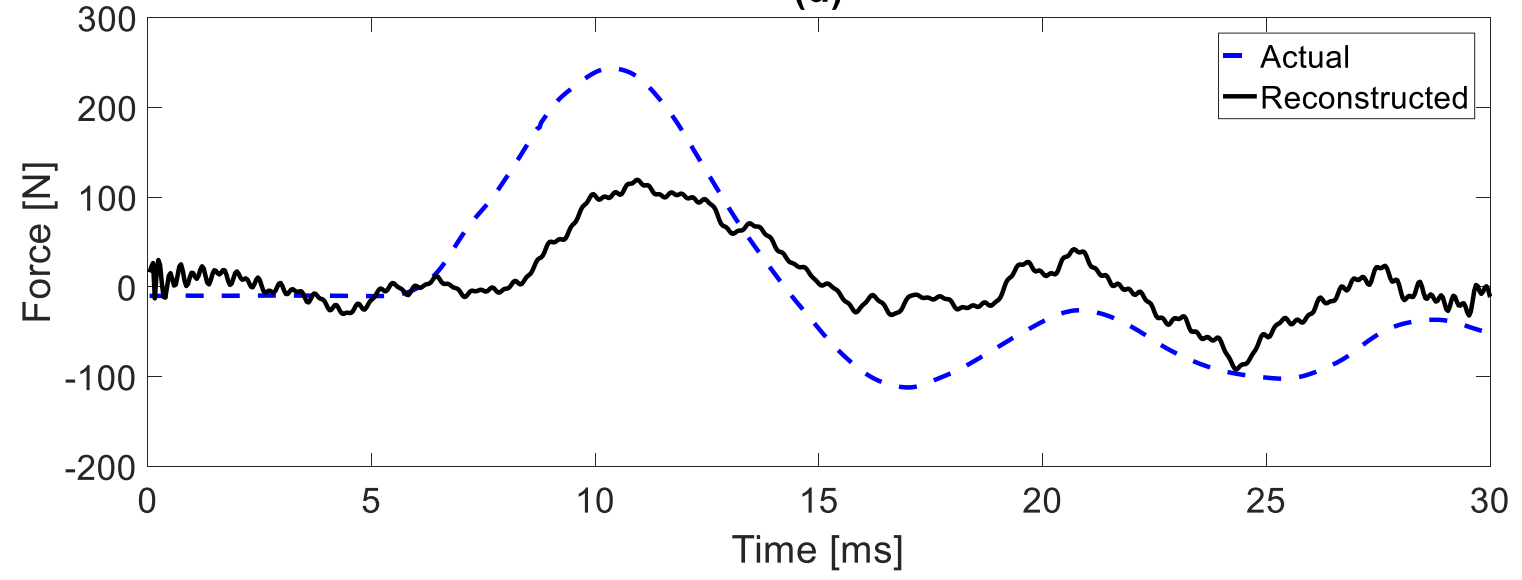

Figure 14. Reconstructed impact forces at (a) location 1, (b) location 2, (c) location 3 and (d) location 4 using velocity responses measured at points 4, 6, 10 and 12 . 
(a)

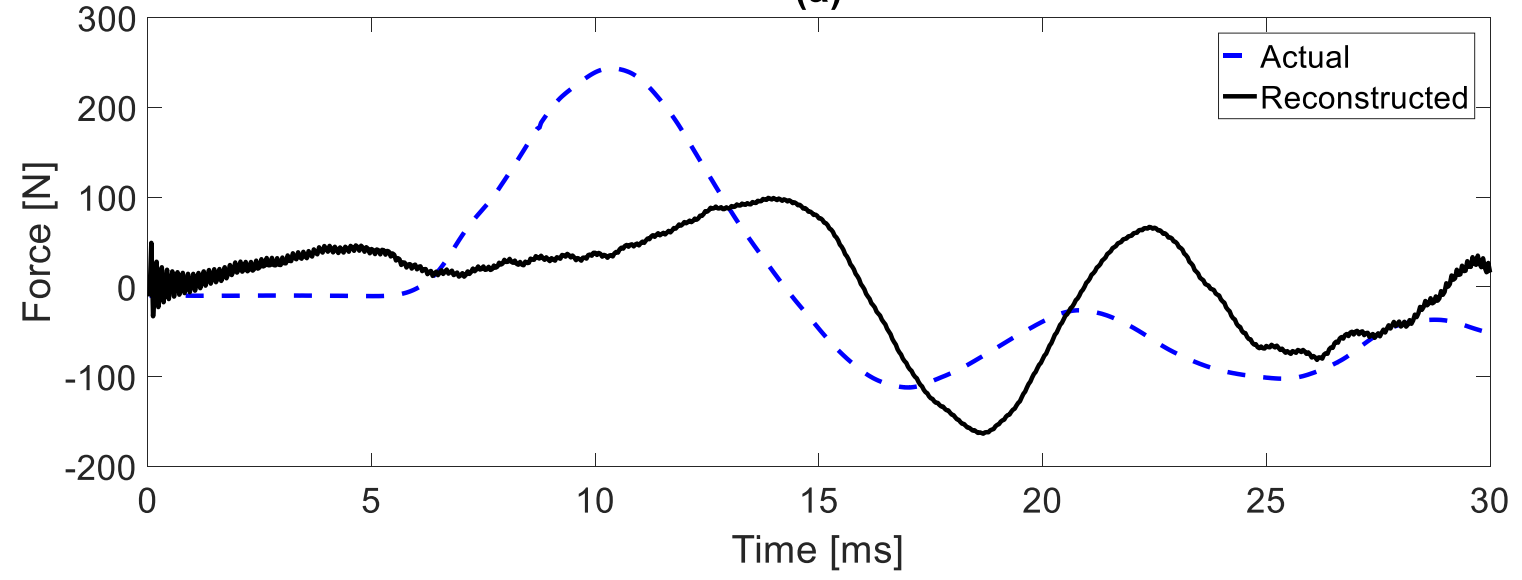

(b)

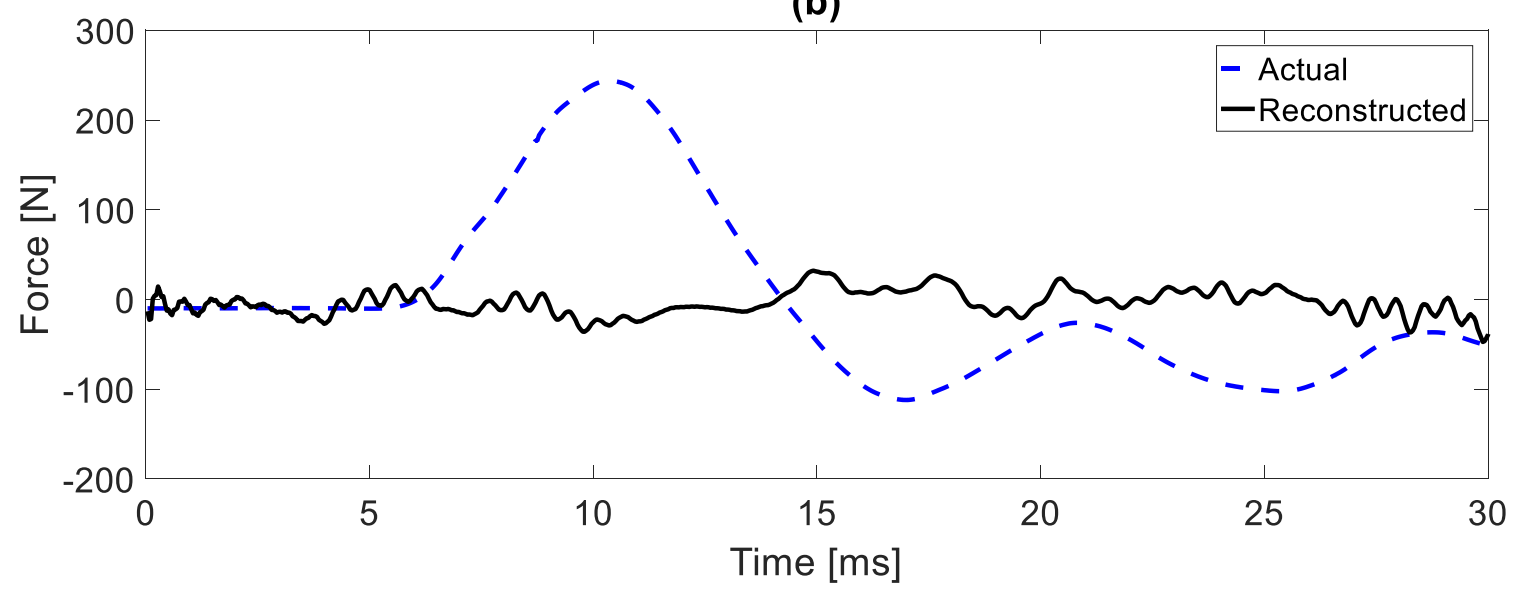

(c)

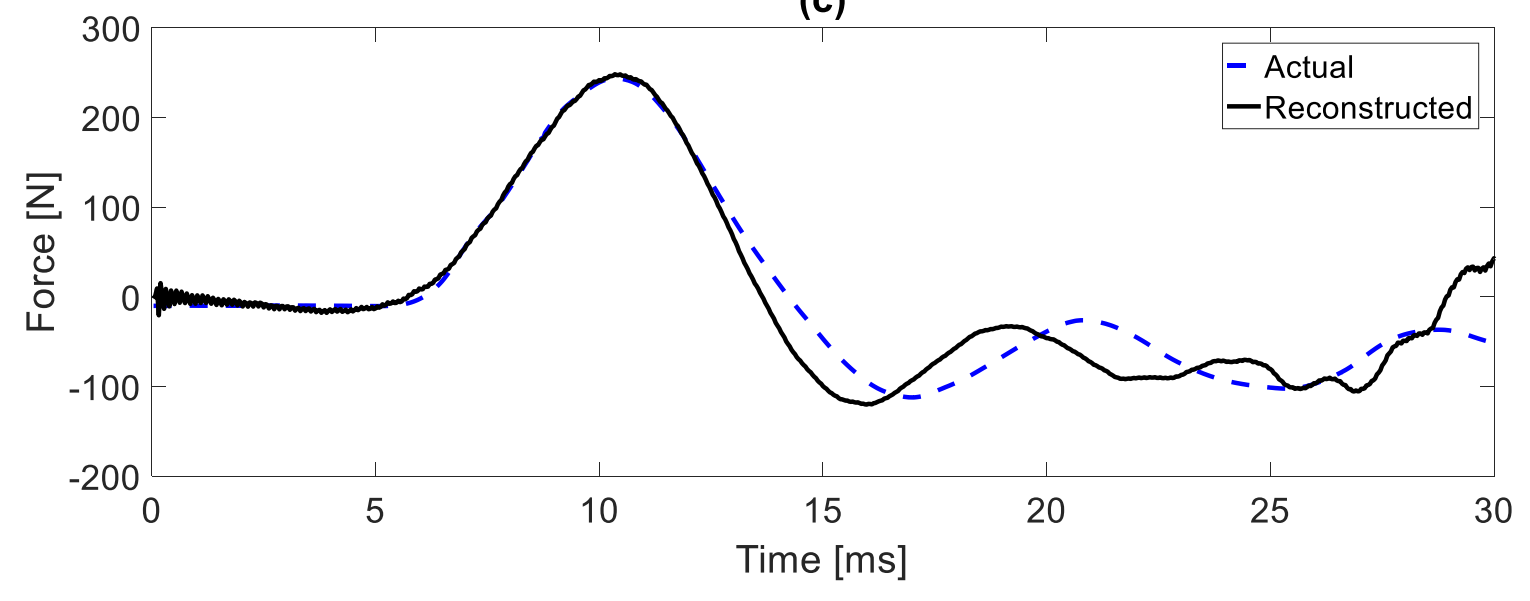


(d)

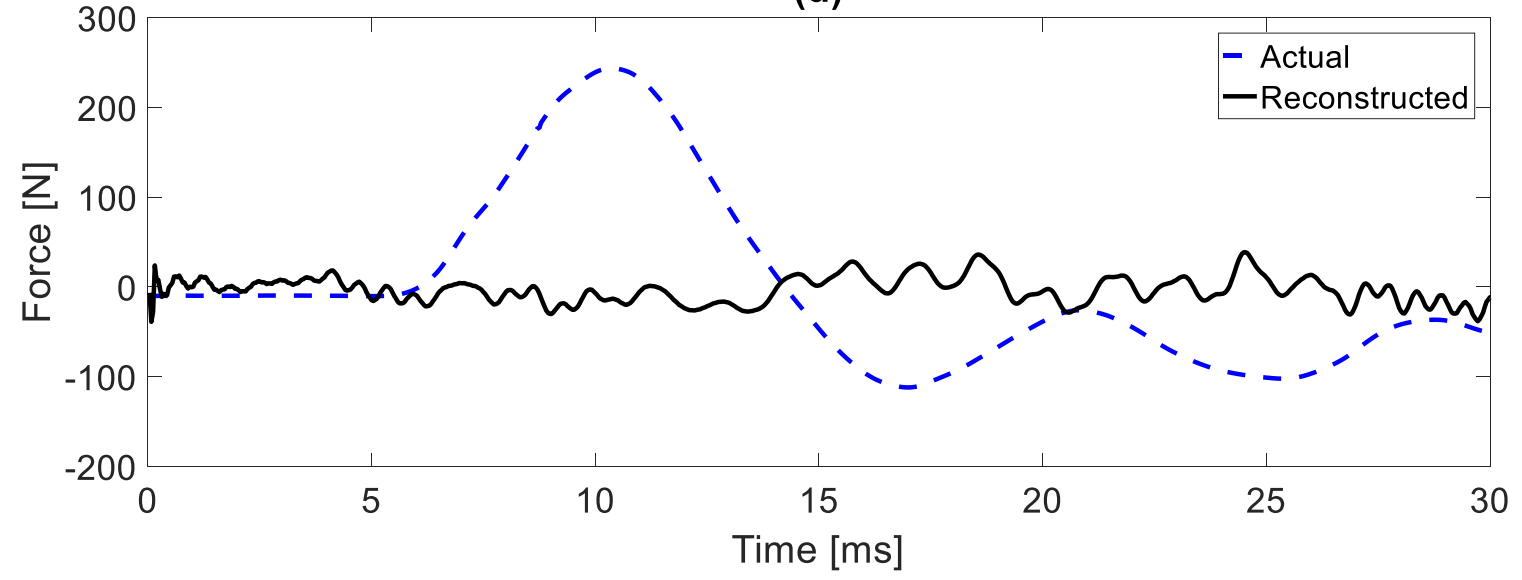

Figure 15. Reconstructed impact forces at (a) location 1, (b) location 2, (c) location 3 and (d) location 4 using velocity responses measured at points $3,4,7$ and 8 . 
(a)
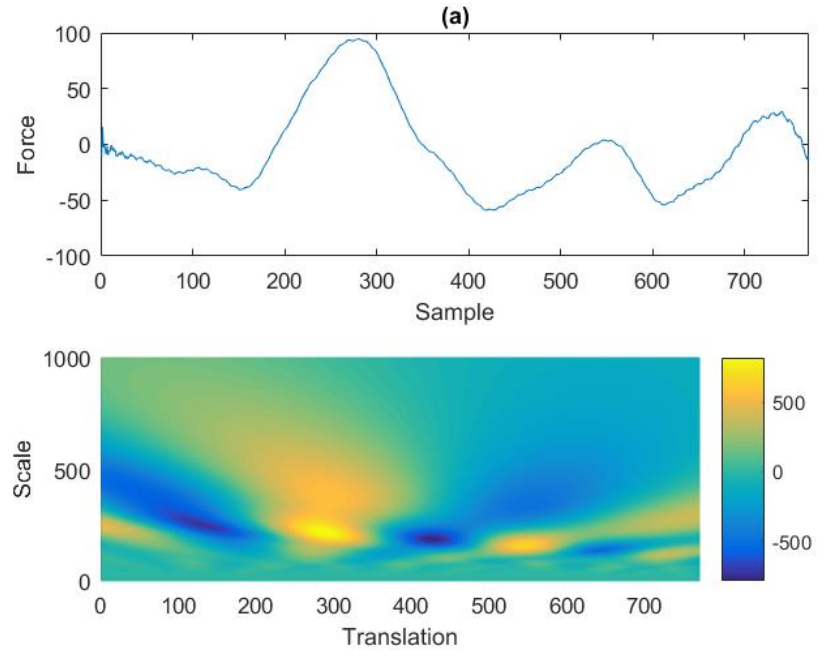

(c)
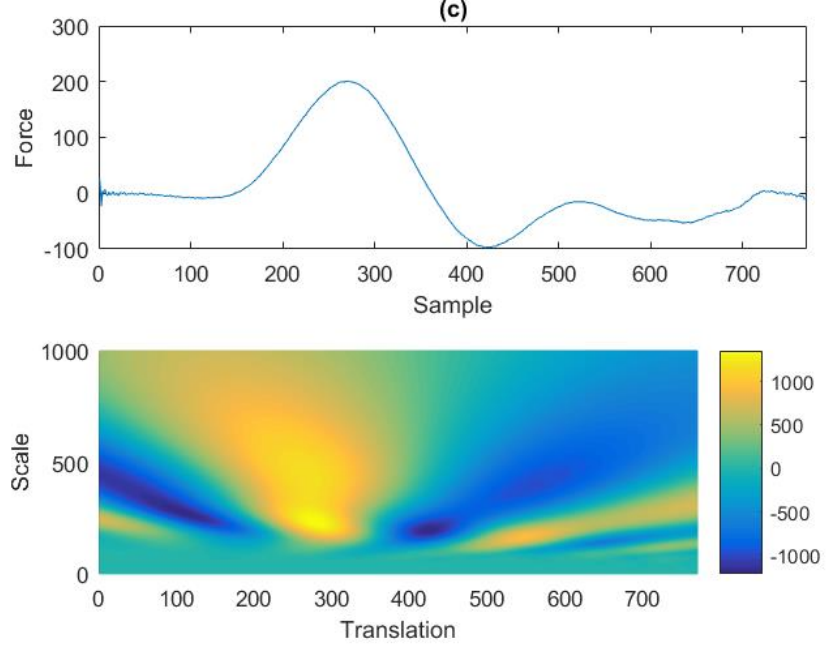

(b)
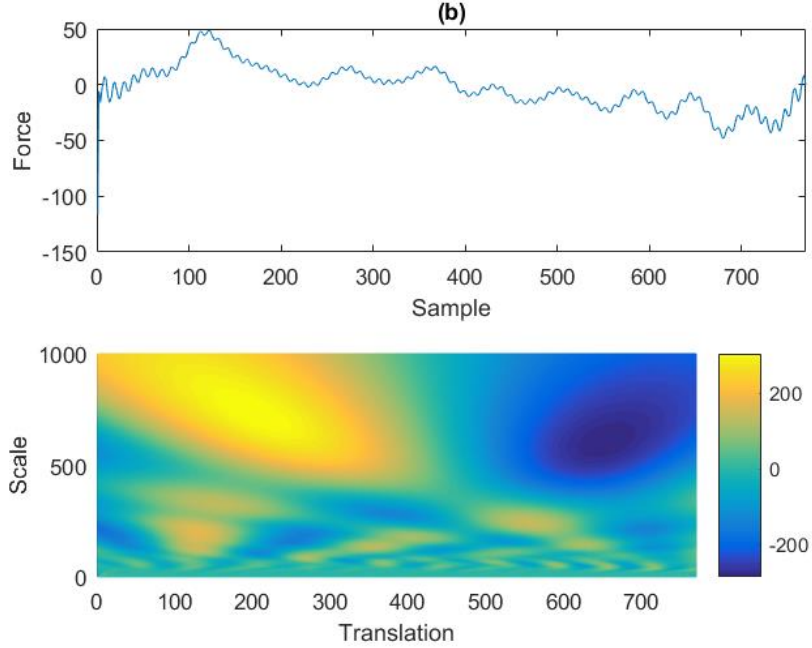

(d)
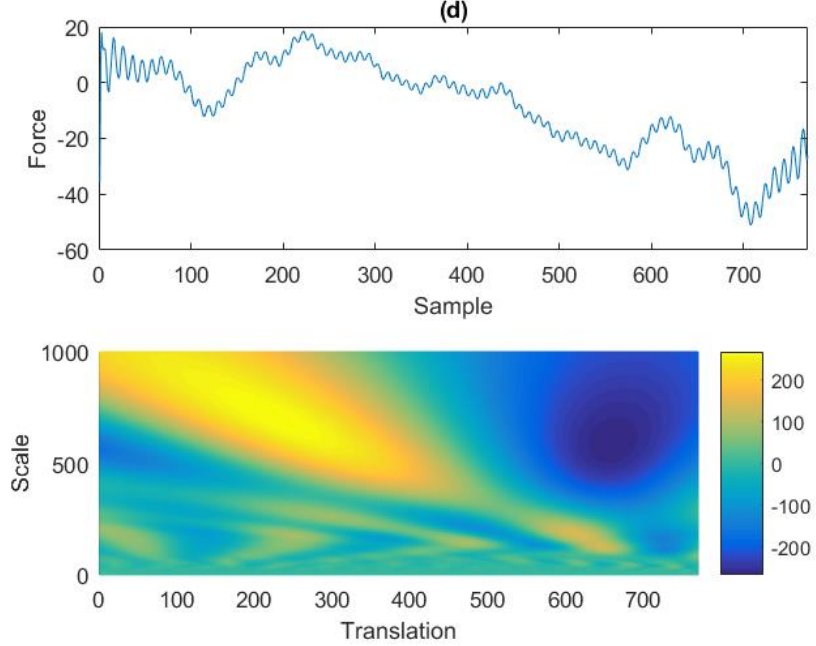

(e)

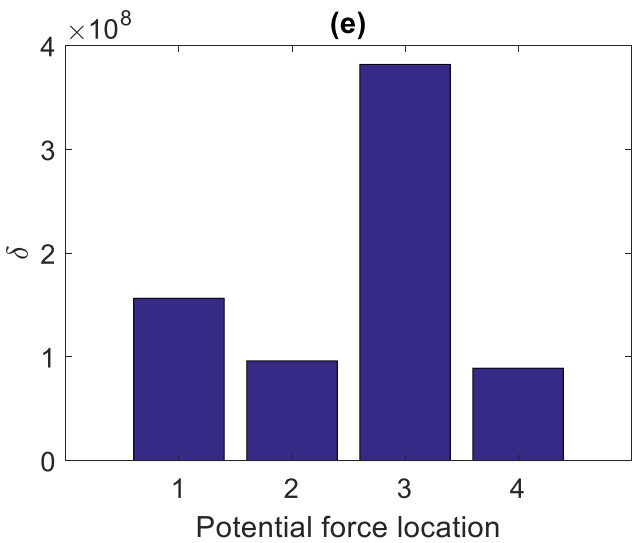

Figure 16. Wavelet coefficients for the reconstructed impact forces shown in Figure 10, (a) location 1, (b) location 2, (c) location 3 and (d) location 4 and (e) the impact force identification index. 
(a)
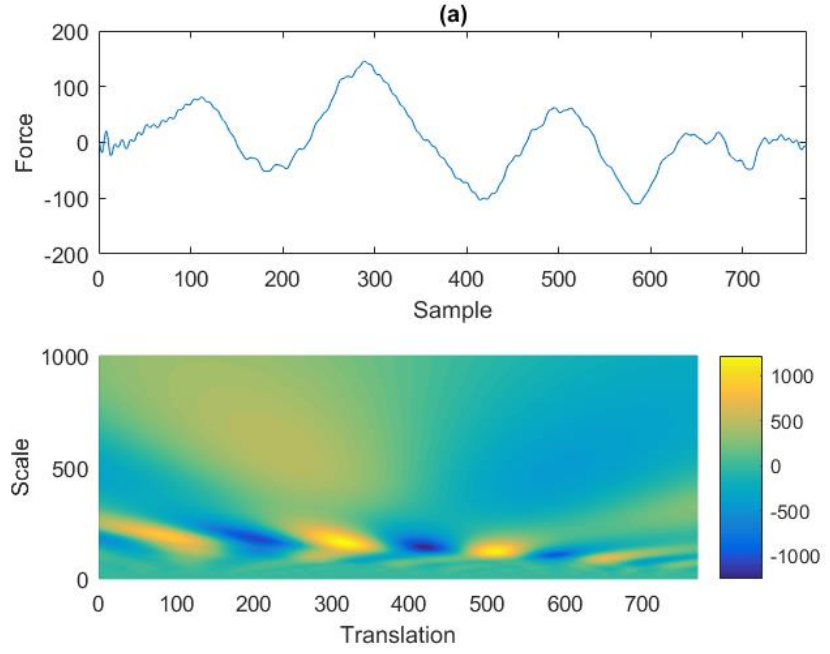

(c)
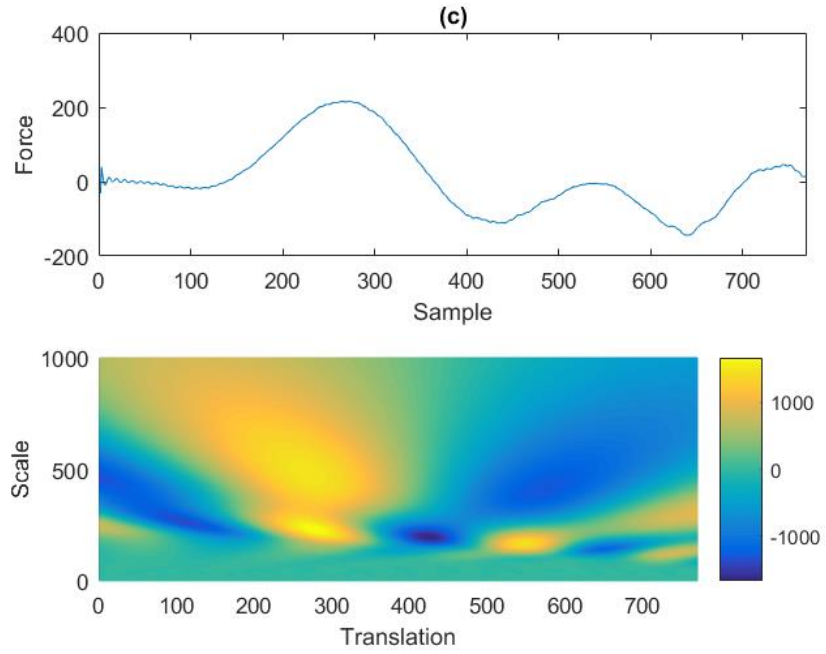
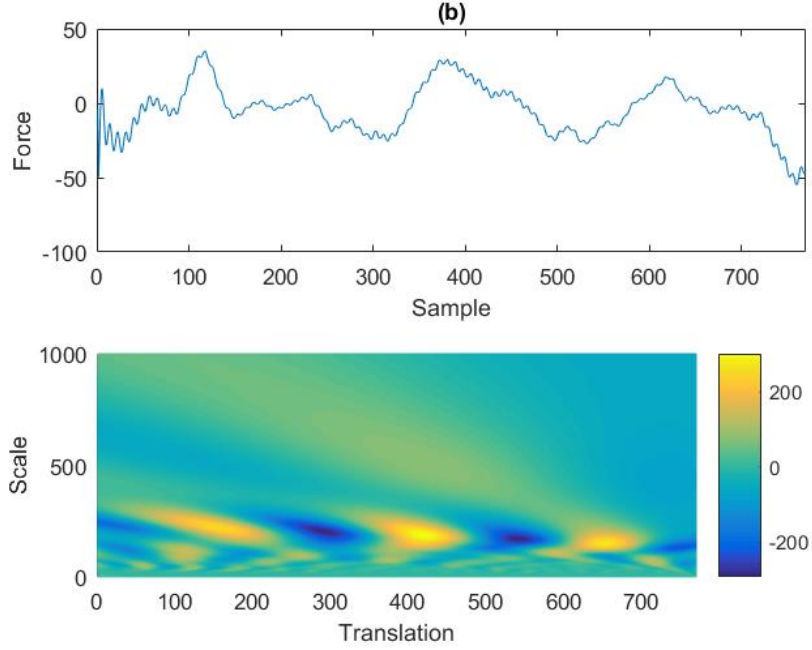

(d)
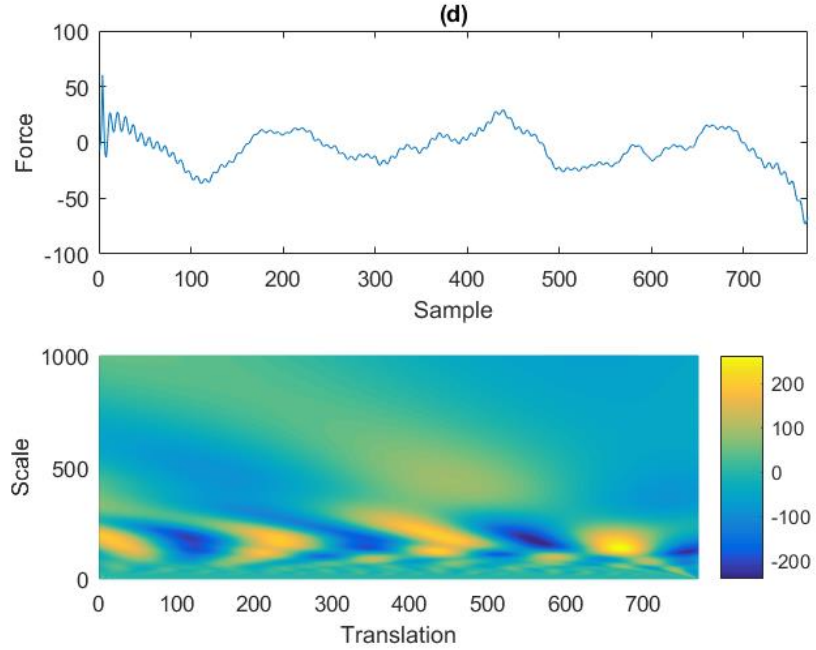

(e)

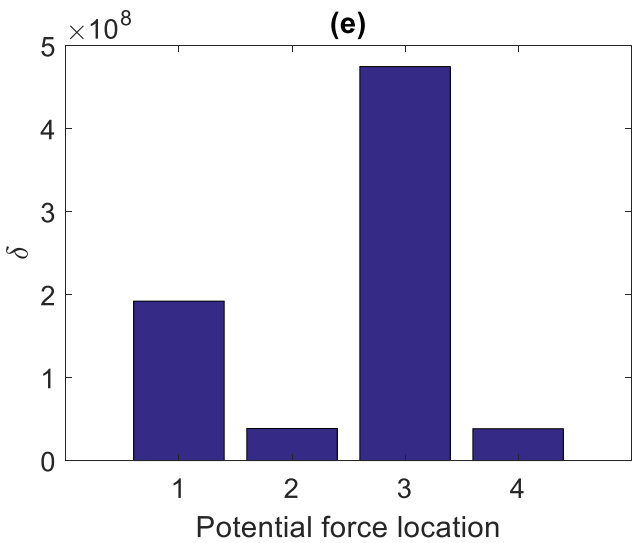

Figure 17. Wavelet coefficients for the reconstructed impact forces shown in Figure 12, (a) location 1, (b) location 2, (c) location 3 and (d) location 4 and (e) the impact force identification index. 

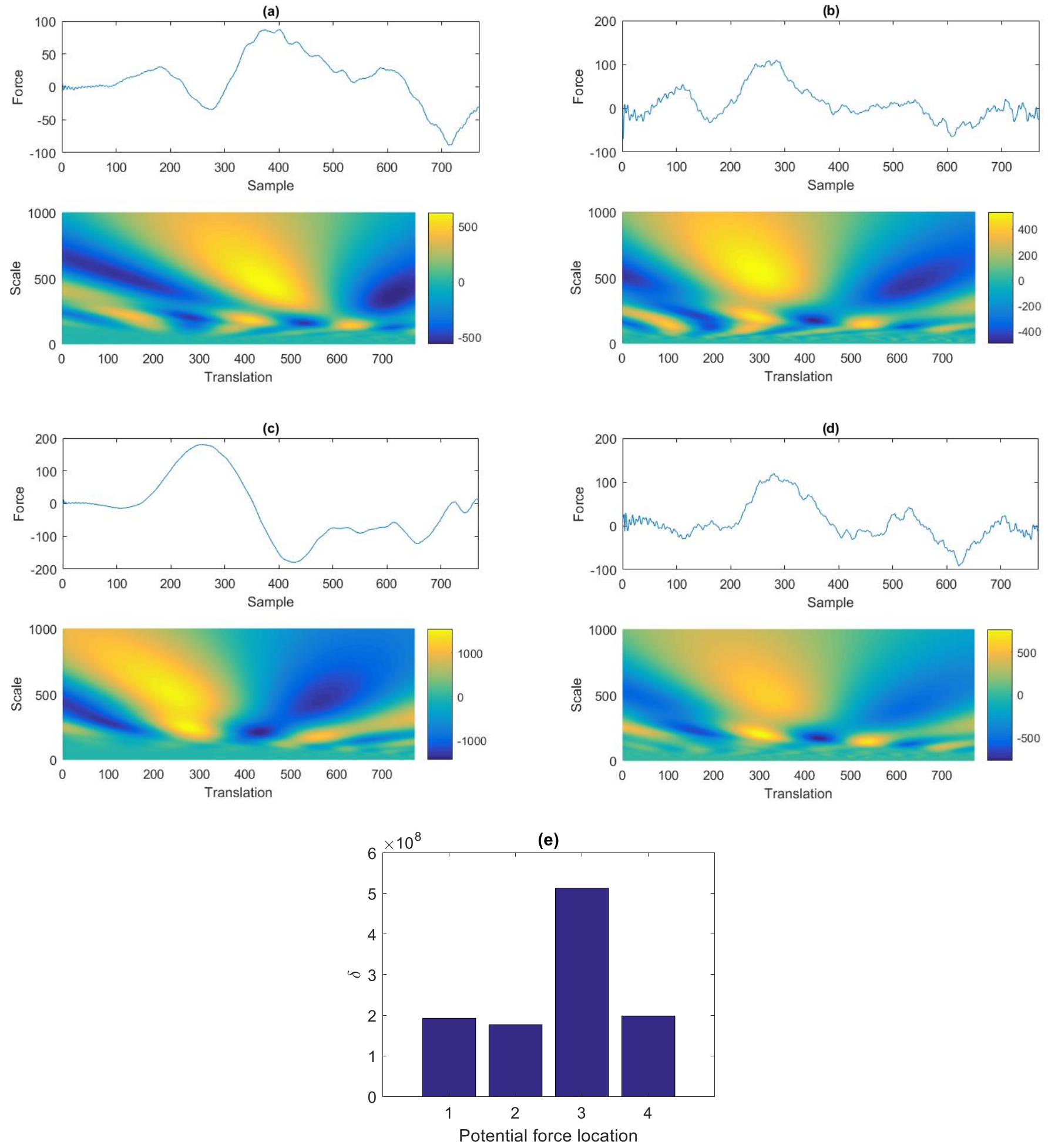

Figure 18. Wavelet coefficients for the reconstructed impact forces shown in Figure 14, (a) location 1, (b) location 2, (c) location 3 and (d) location 4 and (e) the impact force identification index. 

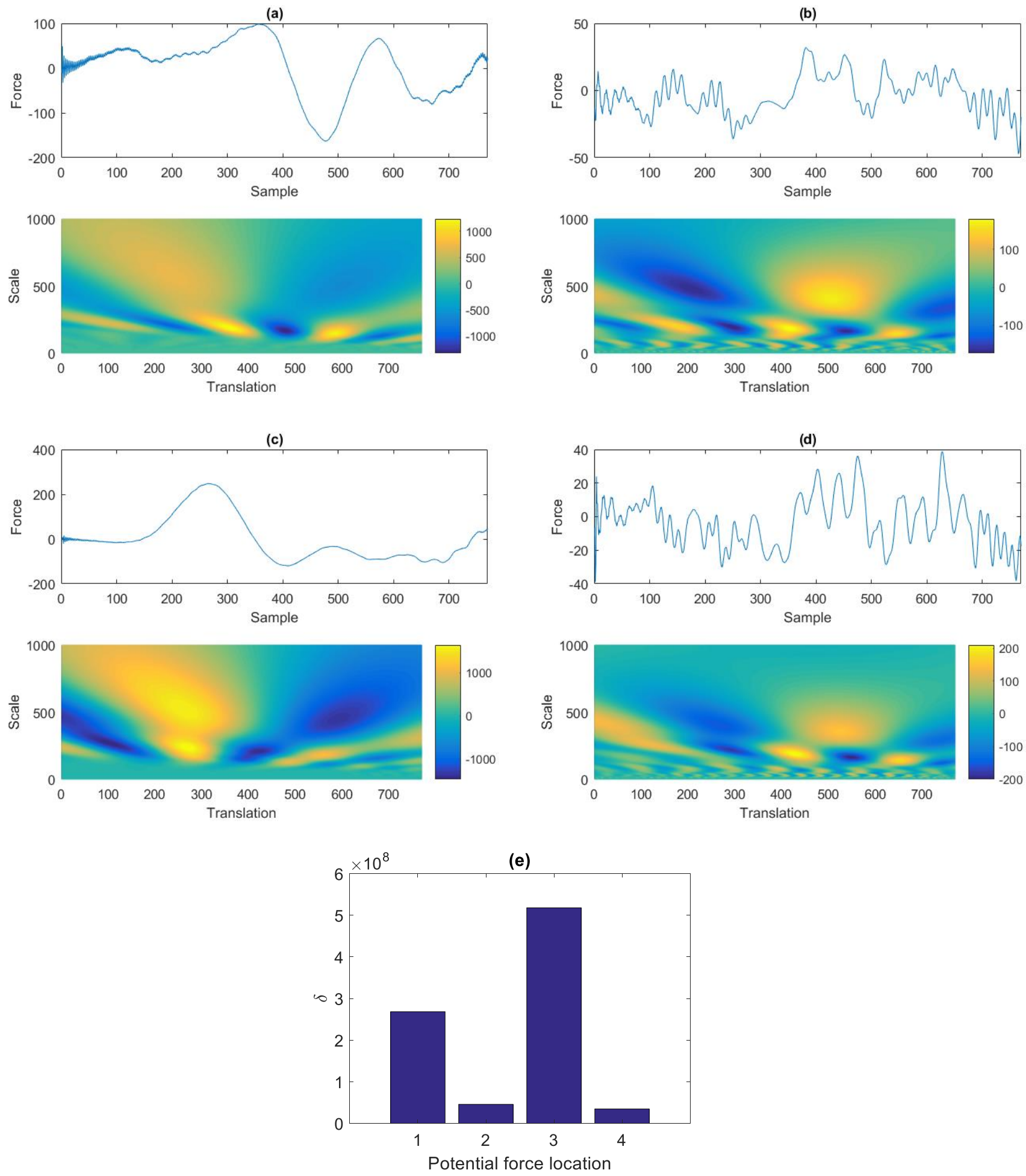

Figure 19. Wavelet coefficients for the reconstructed impact forces shown in Figure 15, (a) location 1, (b) location 2, (c) location 3 and (d) location 4 and (e) the impact force identification index. 